\title{
Type I interferons link skin-associated dysbiotic commensal bacteria to pathogenic inflammation and angiogenesis in rosacea
}

\section{Alessio Mylonas ( $\square$ alessio.mylonas@epfl.ch )}

Swiss Federal Institute of Technology Lausanne (EPFL) https://orcid.org/0000-0001-6220-1973

Heike Hawerkamp

Dusseldorf University Hospital

\section{Yichen Wang}

INSERM UMR 1163, Institut IMAGINE

\section{Olivier Demaria}

University Hospital CHUV

\section{Stephan Meller}

Department of Dermatology, Medical Faculty, Heinrich-Heine-University, Duesseldorf

\section{Bernhard Homey}

Department of Dermatology, Heinrich-Heine-University

Jeremy Di Domizio

University Hospital CHUV https://orcid.org/0000-0002-6281-3918

\section{Lucia Mazzolai}

Univerity Hospital CHUV

\section{Alain Hovnanian}

University Paris Descartes - Sorbonne Paris Cité

\section{Michel Gilliet}

Department of Dermatology, University Hospital of Lausanne (CHUV) https://orcid.org/0000-00024609-3762

\section{Conrad Curdin}

Department of Dermatology, University Hospital of Lausanne (CHUV) https://orcid.org/0000-00022547-3440

\section{Article}

Keywords: Inflammatory Skin Disease, Neovascularization, Microbial Dysbiosis, Plasmacytoid Dendritic Cells, Cathelicidin, Neoangiogenesis

Posted Date: February 26th, 2021 
DOI: https://doi.org/10.21203/rs.3.rs-279140/v1

License: (c) (1) This work is licensed under a Creative Commons Attribution 4.0 International License. Read Full License 


\section{Abstract}

Rosacea is a common chronic inflammatory skin disease that is characterized by a fluctuating course of excessive inflammation and apparent neovascularization. Microbial dysbiosis with high density of B. oleronius and increased activity of the serine protease kallikrein 5 , which cleaves cathelicidin antimicrobial peptide, have been recognized as key pathogenic triggers in rosacea. However, how these events are linked to the hallmarks of the disease remains unknown. Here, we show that type I interferons produced by plasmacytoid dendritic cells represent the pivotal link between dysbiosis, an aberrant immune response, and neovascularization in rosacea. In fact, compared to other commensal bacteria, $B$. oleronius is highly susceptible and preferentially killed by cathelicidin antimicrobial peptides leading to enhanced generation of complexes with DNA. DNA from skin-associated microbiota but not from host cells is required for cathelicidin-induced activation of plasmacytoid dendritic cells and type l-interferon production, which is further amplified by $B$. oleronius. Moreover, kallikrein 5 cleaves cathelicidin into peptides with heightened DNA binding and type I interferon-inducing capacities, further facilitating type I interferon production within the skin. In turn, type I interferons induce IL22 whilst simultaneously rendering endothelial cells responsive through upregulation of the IL22-receptor, and thereby driving drive neoangiogenesis. These findings unravel novel pathomechanisms, which directly link several hallmarks of rosacea to the killing of dysbiotic commensal bacteria and the induction of a pathogenic type-I interferon- $T_{H} 17 / 22$ pathway.

\section{One Sentence Summary}

Flare-ups of rosacea are driven by type I interferon production triggered by commensal bacteria, and result in pathogenic vascular angiogenesis via synergy with IL22.

\section{Introduction}

Rosacea is a common chronic inflammatory disorder mainly affecting the centro-facial skin. It is characterised by a variety of clinical manifestations including transient and persistent erythema, flushing and telangiectasia, papules, pustules, ocular involvement and in some cases phymata. Rosacea often fluctuates between periods of exacerbation and remission. Known trigger factors include temperature changes, UV exposure, emotional stress, exercising, and intake of spicy food or alcohol. More than $5 \%$ of the world's population is affected ${ }^{1}$ though the prevalence varies considerably depending on age, gender, geography, and ethnicity. Due to its conspicuous nature, rosacea can lead to stigmatization and is often accompanied by depression and anxiety. The psychosocial burden can be debilitating and rosacea has a significant impact on the emotional, social, and overall well-being of patients. ${ }^{2,3}$

Accumulating evidence suggest a multifactorial aetiology with microbial dysbiosis, an aberrant response of the innate immune system, and neurovascular dysregulation playing an important role. Demodex mites, which show a higher density in rosacea patients, ${ }^{4-6}$ harbour Bacillus oleronius, a commensal bacterium that has been associated with disease activity in rosacea. ${ }^{7-9}$ The recognition of microbial 
products by pattern recognition receptors, such as toll-like receptor (TLR)-2, which is overexpressed in rosacea, ${ }^{10}$ induce expression of cathelicidin by keratinocytes. ${ }^{11}$ Cathelicidin is an anti-microbial peptide that necessitates proteolytic processing for activation. Kallikrein 5 (KLK5) represents the predominant protease responsible for cleaving cathelicidin and shows increased expression in rosacea. ${ }^{12}$ Likewise, both cathelicidin and its processed active forms, including LL-37, FR-29, FA-29 and DI-27, are also overexpressed in lesional skin of rosacea patients. ${ }^{13} \mathrm{LL}-37$ has been shown to induce inflammation and oedema, drive chemotaxis of various immune cells, and promote angiogenesis, which correlate with characteristics of rosacea ${ }^{13-15}$ However, the exact pathogenic role of these antimicrobial peptides remains to be elucidated for rosacea.

It is well established, that LL-37 is able to bind and condense nucleic acids, which are released by dying host cells upon injury, and allows the internalisation of these complexes into plasmacytoid dendritic cells (pDCs). Thereby, LL-37 enables activation of endosomal TLR-7 and 9 and potent induction of type I interferons (IFN) by pDCs. ${ }^{16,17}$ Whereas upon skin injury, regulated LL-37 expression leads to transient type I IFN production and protective immune responses, ${ }^{18}$ overexpression in psoriasis drives excessive pDC activation resulting in IFN-driven autoimmunity. ${ }^{19-21}$

Despite recent scientific advances, the pathomechanisms underlying rosacea are still poorly understood and current treatment options remain mostly broad and symptomatic. Particularly little is known about early instigators of exacerbations and recurring flare-ups, which would allow development of more targeted, preventive treatment options.

Here, we report that pDC-derived type I IFNs are overexpressed selectively in acute flare-ups of rosacea. In vivo blockade of type I IFN receptor (IFNAR) signalling and $\mathrm{pDC}$ depletion both result in a loss of the TH22-/TH17-immune response that is characteristic for rosacea. We show that skin commensal bacteria are necessary and the rosacea-associated bacterium $B$. oleronius is sufficient for the induction of a pathogenic type I IFN-driven immune response by LL-37 in vivo. Moreover, kallikrein 5 cleaves cathelicidin into peptides with heightened DNA binding and type I interferon-inducing capacities, which further facilitate type I interferon production. Finally, type I IFN and IL22 cooperate in the induction of vascular endothelial cell (EC) survival and proliferation, both in vitro and in vivo, giving rise to aberrant angiogenesis. Thus, several rosacea hallmarks can be directly linked to the killing of dysbiotic commensal bacteria, which initiate type I IFN-driven flare-ups and IL22 mediated neoangiogenesis, confirming a pathogenic functional role for this pathway in rosacea.

\section{Results}

Innate and adaptive cytokines associated with $T_{H} 1 / 17 / 22$, but not $T_{H} 2$, are overexpressed in rosacea

In order to identify potential early instigators of rosacea skin inflammation, we analysed skin biopsies from rosacea patients taken either during acute flare-ups or during stabilised chronic inflammation (Suppl. Table 1) and compared them to skin from healthy donors. 
First, we confirmed overexpression of the cathelicidin transcript hCAP18 in rosacea (Suppl. Fig. 1a), as described in a previous report. ${ }^{13}$ We then analysed mRNA expression levels of selected innate and adaptive cytokines involved in the pathogenesis of different inflammatory skin diseases to identify unique expression patterns. Among innate cytokines, we observed a significant increase in $T N F, I L 1 B, I L 8$, $I L 12 B, I L 23 A$, and $I L 10$ but not $I L 36 B$ in rosacea skin lesions as compared to skin from healthy donors (Suppl. Fig. 1a and Fig. 1a). Furthermore, we could observe a trend increase in IL6 expression, and bimodal distribution for IFNA2 and IFNB1. Adaptive T cell-derived cytokines IFNG, IL17A, IL17F, and IL22 were significantly overexpressed in lesional rosacea skin while IL 4 and $I L 13$ were either undetectable or not increased (Suppl. Fig. 1b and Fig.1b). These results are in line with previously published data ${ }^{22}$ indicating a predominant $T_{H} 1 / T_{H}$ 17-related inflammation in rosacea. When comparing acute flare-ups with stabilized chronic lesions of rosacea in order to identify potentially unique patterns, we found a selective and significant increase in expression of type I IFNs IFNA2 and IFNB1 in flare-ups (Fig.1a). Interestingly, whereas type I IFNs were selectively upregulated in rosacea patients during acute flares, the type I IFN response gene $M X 1$ was upregulated in all rosacea samples as compared to healthy skin (Suppl. Fig. 1C and Fig. 1c) suggesting that type I IFNs are transiently produced early during acute flareups of rosacea. All other cytokines revealed comparable expression levels between acute flare-ups and chronic stabilized lesions though there was a tendency for an increased expression of TNF in more chronic rosacea lesions (Fig.1a and 1b).

Taken together, these findings indicate the following: first, rosacea is characterised by predominant $\mathrm{T}_{\mathrm{H}} 1 / \mathrm{T}_{\mathrm{H}} 17$ signatures, and second, rosacea is driven by early overexpression of type I IFN during acute flare-ups, which then is subsequently relayed by TNF and a chronic inflammation.

\section{Type I interferon overexpression correlates with pDC infiltration in flares of rosacea}

As type I IFNs are preferentially expressed by pDCs, we investigated their presence in rosacea by staining paraffin-embedded sections for CD123 (IL3RA). Lymphoid cells with strong CD123 expression represent bona-fide pDCs as shown by co-expression of BDCA2 and absence of CD11c by microscopy (Fig. 2a). While largely absent in facial skin of healthy donors, we found high numbers of pDCs within the dermal infiltrate of rosacea (Fig 2b). When plotting the percentages of $\mathrm{CD}_{123^{+}}$pDCs (Fig. 2b) along with IFNA2 (Fig.1a) and IFNB1 expression (not shown) we found statistically significant monotonic positive correlation between the two (Fig. 2d; IFNA2: $r=0.7363, p=0.001$; IFNB1: $r=0.7951, p=0.0002$ ), indicating that $\mathrm{pDC}$ represent the principal source of type I IFNs during flare-ups of rosacea.

Type I interferon expression is critically dependent on pDCs in a mouse model of rosacea

To investigate this functionally, we took advantage of a previously described in vivo model of rosacea. ${ }^{13}$ Briefly, the cathelicidin antimicrobial peptide LL-37, which is overexpressed in skin of rosacea patients 
(Suppl. Fig 1 ; and ${ }^{13}$ ), is injected intradermally four consecutive times over a period of $48 \mathrm{~h}$. We found that, upon injection of LL-37, pDCs are rapidly recruited into the skin and accumulate over time (Fig. 3a). The pDC accumulation significantly correlated with gene expression of Ifna $1(r=0.7056, p>0.0001)$ and Ifnb1 $(r=0.6784, p<0.0001$; Fig $3 b)$. Moreover, depletion of pDCs by anti-Pdca1 antibodies completely abolished Ifna 1 expression and largely abrogated Ifnb1 expression in the skin (Fig. 3c). Congruently, induction of downstream type I IFN stimulated genes $M \times 2$ was significantly reduced and entirely abolished for Isg 15 and Irf7 (Suppl. Fig. 2a). We obtained similar results using a different pDC depletion system, the BDCA2DTR transgenic mouse (Suppl. Fig. 2), confirming in vivo that pDCs are the principal source of type I IFNs. Interestingly, blocking type I IFN signalling by an anti-Ifnar1 antibody not only blocked type I IFN signature genes but also significantly reduced the numbers of pDCs infiltrating the skin (Fig. 3d). These results indicate that the continuous overexpression of LL-37 in rosacea increases type I IFN production by pDCs, which further amplifies their skin infiltration and sustains in situ production of type I IFNs. Indeed, Cxcr3family chemokines $\mathrm{Cxcl} 9, \mathrm{Cxcl} 10$, and $\mathrm{Cxcl} 11$, which mediate pDC migration into the skin, were induced by LL37, and their expression was highly dependent on pDCs and Ifnar signalling (Suppl. Fig 3).

\section{Type I interferon blockade and pDC depletion abolish select $T_{H} 1 / T_{H} 22$ signatures}

Next, we sought to analyse the functional role of pDC-derived type I IFNs in driving pathogenic inflammation in rosacea. First, we investigated if the in situ cytokine expression profile in the mouse model indeed reflects human rosacea. The expression of pro-inflammatory innate cytokines, including $\mathrm{T}_{\mathrm{H}}$ 1- polarising cytokines $/ / 12 a$ and $T n f, \mathrm{~T}_{\mathrm{H}} 17-/ 22$-polarising cytokines $/ / 1 b, \| / 23 a$, and $/ / 6$, as well as $/ / 10$ were significantly induced as compared to controls showing a similar expression profile as in rosacea (Fig.4a). Overexpression of Tnf, I/1b, I/23a, and I/6 was critically dependent on pDCs and type I IFN signalling, while induction of $/ / 36 \mathrm{~b}$ and $/ / 10$ expression was independent of either. All adaptive cytokines tested, including $/ / 17 a, I / 17 f$ and $/ / 22, I / 4$ and $/ / 13$, and Ifng, were significantly induced upon LL-37 injection. However, only the overexpression of $/ / 17 f$ and $I / 22$, but not of $T_{H} 2$ and $T_{H} 1$ family cytokines, was dependent on pDCs and type I IFN signalling (Fig. 4b). These findings indicate that LL-37 overexpression in rosacea activates skin-infiltrating pDCs to produce type I IFN, which is required to drive a $T_{H}$ 17-/22-related inflammation.

Cathelicidin peptides found in rosacea display differential potencies to bind and complex DNA and to induce type I interferons

It is widely established that LL-37 is able to activate pDCs to produce large amounts of type I IFNs by internalising extracellular nucleic acids. In rosacea, however, cathelicidin is processed into several additional C-terminal peptides besides LL-37, such as FR-29, FA-29, and DI-27 (Suppl. Fig. 4; and ${ }^{13}$ ). These, despite considerable overlap (Suppl. Fig. 4b) display significant differences in terms of 
hydrophobic surfaces (Suppl. Fig. 4a-c), charge, and percentage of predicted alpha-helical structures (Suppl. Fig. 4d). Thus, we wondered whether these peptides had a differential ability to activate pDCs. We isolated human pDCs and stimulated them with complexes of DNA and the different cathelicidin derived peptides. When complexed with DNA, FR-29 showed significantly increased IFNa production by pDCs as compared to LL-37 for the same molar concentration, (Fig.5a). Neither FA-29 nor DI-27 were able to stimulate detectable amounts of IFNa from pDCs in vitro. When reducing the concentration of peptides below the stimulatory capability of LL-37 to $3 \mu \mathrm{M}$, FR-29 retained significant - albeit reduced stimulatory capacity. To analyse the underlying mechanism of differential IFNa production, we first assessed the DNA-binding capacity of these peptides by picogreen fluorescence quenching. Indeed, FR-29 bound and condensed DNA more potently than LL-37 (Suppl. Fig. 5). Conversely, FA-29 and DI-27 had almost indiscernible DNA binding activity. To further assess the capacity of FR-29 and LL-37 to internalise DNA into pDCs, we fluorescently tagged purified, undigested DNA, and complexed it with the cathelicidin peptides LL-37 and FR-29. Whereas PDCs were unable to uptake DNA in the absence of cathelicidin peptides, LL-37 and FR-29 both showed uptake of fluorescently labelled DNA by ca. $10 \%$ of cells (Fig.5b). However, the relative uptake of DNA was markedly more prominent when complexed with FR-29 than with LL-37 as exemplified by the increased fluorescence intensity. Taken together, these data indicate that several fragments found in rosacea such as FA-29 and DI-27 cathelicidin peptides fail to activate pDCs, whereas FR-29 has a significantly more potent effect than LL-37 due to increased affinity to nucleic acids.

To further evaluate the in vivo relevance of these findings, we analysed Ifna gene expression in the rosacea mouse model. Indeed, intradermal injection of FR-29 induced significantly more potent Ifna1 production than LL-37 (Fig.5c). pDC infiltration of the skin was comparable between FR-29 and LL-37 suggesting that enhanced Ifna1 production upon FR-29 injection is due to a differential activatory capacity of the cathelicidin peptides rather than increased skin infiltration by pDCs (Fig.5d).

Kallikrein 5 recapitulates $p D C$-dependent type I interferon expression

Cathelicidin pro-peptide processing is required for LL-37 activity in the skin. Kallikrein 5 (KLK5), which is overexpressed in rosacea, ${ }^{10,13}$ mediates processing of cathelicidin into the active LL-37 peptide. To determine whether KLK5 overexpression is sufficient to instigate activation of the LL-37/pDC/type I IFN axis, we took advantage of a recently described transgenic murine model expressing human KLK5 in the epidermis under the $\mathrm{K} 5$-promoter $\left(\mathrm{Tg} . K L K 5\right.$; and $\left.{ }^{23}\right)$. First, we confirmed in vitro and in vivo that human KLK5 is able to cleave Cramp pro-peptide, the murine analogue of human cathelicidin. In vitro, human KLK5 effectively processed murine Cramp pro-peptide as shown by western blot (Fig. 6a). In addition, spontaneously after birth, we found significantly increased expression of Cramp pro-peptide and increased presence of the cleaved active form of Cramp in the skin of Tg.KLK5 mice as compared to wild type littermates (Fig. 6b). These findings indicate that constitutive overexpression of KLK5 in the skin leads to accumulation of active cathelicidin peptides similar as seen in rosacea. Next, we investigated the 
presence of pDCs in skin of these mice. Seven days after birth, pDCs accumulated significantly and spontaneously in Tg.KLK5 mice but not control mice (Fig. 6c), and were paralleled by type I IFN overexpression (Fig. 6d). Whereas type I IFNs were upregulated early in the skin and declined over time, $\mathrm{T}_{\mathrm{H}}$ 17-related cytokines $/ / 22, \| 17 \mathrm{a}$, and $/ / 17 f$ were significantly overexpressed only at a later time point indicating that type I IFNs might drive $T_{H} 17$ response in Tg.KLK5 mice. Depletion of pDCs from birth led to loss of induction of type I IFN in the skin, confirming pDCs as the principal source of type I IFN in the Tg.KLK5 mouse model (Fig. 6e). Furthermore, blockade of type I IFN signalling significantly inhibited expression of $T_{H} 17 / T_{H} 22$ cytokines $/ / 22$ and $/ / 17 f$, in line with data obtained in the LL-37 intradermal injection model (Fig. 6f). Taken together, these findings indicate that KLK5 overexpression in rosacea increases cleavage of cathelicidin in the skin. In turn, accumulating active cathelicidin peptides attract and activate pDCs resulting in a type I IFN-driven $T_{H} 17 / T_{H} 22$ immune response characteristic for rosacea.

\section{Cathelicidin peptides kill bacteria associated with rosacea leading to activation of pDCs}

It has been shown that complexes of host DNA and antimicrobial peptides such as LL-37 play an important role in the pathogenesis of several chronic inflammatory diseases such as psoriasis, lupus erythematosus, or atherosclerosis. ${ }^{16,24,25}$ Because rosacea severity has been linked to dysbiotic communities of commensal skin bacteria, in particular Bacillus oleronius and Staphylococcus epidermidis, we wondered whether commensal microbial DNA rather than host DNA could play a pathogenic role in driving inflammation in rosacea. First, we investigated killing of different skin and nonskin associated bacteria by LL-37. B. oleronius was the most susceptible, followed by the other skinassociated bacteria S. epidermidis and $C$. acnes (Fig. $7 \mathrm{a}$ and b). Gut and lung-associated bacteria, though still subject to killing, were far less susceptible, indicating a preferential killing of skin commensals. When comparing the different rosacea-associated cathelicidin peptides, we found that also FR-29 and FA-29 but not DI-27 were able to kill all bacteria tested (Suppl. Fig. 6). Similar to its ability to condense DNA and activate pDCs, FR-29 showed a higher potency than LL-37 in killing B.oleronius and S.epidermidis, while the latter was slightly more potent or at least equally efficient in killing the other strains of bacteria tested (Suppl. Fig. 6). These data indicate that the different cathelicidin peptides found in skin of rosacea patients show a slightly different spectrum of antimicrobial activity. Moreover, FA-29, which is not able to bind and condense DNA, efficiently killed several strains of bacteria at similar concentrations as LL-37. Thus, the ability of cathelicidin peptides to condense DNA and activate endosomal TLRs is not directly linked to their antimicrobial capacity to kill bacteria.

Next, we tested if LL-37-killed B.oleronius could serve as DNA source and activate pDCs in vitro. We isolated human pDCs from healthy donors and cultured them in presence of live bacteria with or without LL-37. Whereas B.oleronius alone was not able to activate pDCs, the addition of LL-37 allowed for potent activation of pDCs leading to important production of IFNa (Fig. 7c). In accordance with our previous 
results, FR-29 was able to induce significantly more IFNa by pDCs than LL-37 when in the presence of B.oleronius, whereas FA-29 and DI-27 displayed poor capacity to activate pDCs (Fig. 7d).

To further investigate the in vivo relevance of these findings, we took advantage of the rosacea mouse model and injected heat-killed B.oleronius alone or pre-incubated with LL-37. Interestingly, B.oleronius alone was not able to induce type I IFN expression within 24h. Pre-incubated with LL-37 however, B.oleronius induced significantly more type I IFN expression than LL-37 alone (Fig. 7e). These finding indicate that an increased bacterial load alone is not sufficient to rapidly engage the type I IFN pathway but that bacterial killing by cathelicidin peptides is required. As B.oleronius further increased type I IFN expression within the skin upon LL-37 injections, we wondered if commensal skin bacteria are actually a prerequisite to activate pDCs and induce IFNa production in our rosacea model. Thus, we pre-treated mice with a topically applied wide-spectrum antibiotics for 2 days, prior to LL-37 injections. We found that type I IFN expression was almost completely abolished upon antibiotic treatment of the skin (Fig. 7f), whereas injection of B.oleronius with LL-37 was sufficient to restore and enhance type I IFN even further.

Thus, skin commensal bacteria are required but, by themselves, not sufficient to rapidly activate pDCs and induce type I IFN in rosacea. On the other hand, B.oleronius is sufficient for cathelicidin-driven overexpression of type I IFN in the skin. Taken together, these findings indicate a functional role for dysbiotic communities of commensal bacteria in triggering a pathogenic immune response and flare-ups of rosacea.

\section{Type I interferon and IL22 co-operate in the induction of pathogenic angiogenesis}

One of the hallmarks of rosacea is recurrent episodes of flushing and eventually fixed centro-facial erythema, which is caused by neoangiogenesis and telangiectasia. Therefore, we wondered if the KLK5LL-37-pDC-IFNa- $T_{H} 17 / 22$ axis could play a pathogenic role in driving neoangiogenesis in rosacea. First, we assessed the number of dermal ECs as well as their proliferative status in the rosacea mouse model. LL-37 injections significantly increased the number of ECs (Fig. 8a) and the percentage of proliferating ECs (Fig. 8b) as compared to control injections. As FR-29 injections further augmented numbers and proliferation of ECs, we questioned if the pro-angiogenic effect of cathelicidin peptides ${ }^{14}$ might be through activation of the IFNa- $\mathrm{T}_{H}$ 17/22 pathway. Indeed, blockade of type I IFN signalling significantly reduced both the overall numbers and percentage of proliferating ECs (Fig. 8c). To further dissect the mechanism, we sought to determine the effect of IFNa on primary ECs, which are dependent on growth factors for survival and proliferation in culture. While EC growth factors maintained viability and markedly increased cells numbers in vitro, IFNa did not have any discernible direct effect on EC viability and proliferation (Suppl. Fig. 7a-c). So, we tested if type I IFN-induced downstream $T_{H} 17 / 22$ cytokines were instead able to promote EC proliferation. Neither IL17 nor IL22 alone had a direct effect on EC viability. However, ECs cultured in the presence of both IFNa and IL22 showed maintained viability and significantly increased cell numbers. Interestingly, this effect was specific for IL22 as IL17A did not 
augment EC viability when combined with IFNa (Fig. 8d and Suppl. Fig. 7c and 7d). As IFNa is required for IL22-driven EC proliferation, we wondered if IFNa mediated its effect via upregulation of the IL22receptor on ECs. The expression of IL22RA1 - the receptor subunit specific for IL22 - was absent on unstimulated ECs but greatly and significantly induced upon stimulation with IFNa (Fig. 8e). On the other hand, IL17RA and IL17RB, which form the active receptor for IL17, as well as IL10RB, the second subunit of the IL22 receptor, were constitutively expressed and not altered by addition of IFNa or growth factors. These results show that IFNa renders ECs susceptible to IL22 through upregulation of its cognate receptor, thereby enabling IL22-driven proliferation. To verify this in vivo, we assessed EC numbers and proliferation upon IL22-blockade in the rosacea mouse model. Indeed, inhibition of IL22 significantly reduced the numbers and percentage of proliferating ECs in the skin (Fig. 8h) similar to type I IFN blockade (Fig 8c). LL-37 injections led to neoangiogenesis and formation of visible microvessels, as assessed by in vivo videocapillaroscopy, which resembled images taken from human rosacea patients (Suppl. Fig. 7e). These microvessels were significantly reduced upon Ifnar blockade and inhibition of II22 (Fig. 8g). In summary, overexpression of cathelicidins induces vascular EC proliferation and neoangiogenesis in rosacea via induction of pDC-derived type I IFN, which in turns leads to upregulation of IL22 and expression of the IL22-receptor on EC thereby enabling IL22-driven neoangiogenesis.

\section{Discussion}

Our study identifies key pathogenic mechanisms driving acute flare-ups of rosacea. We found transient overexpression of type I IFN in acute rosacea correlating with accumulating pDCs in the dermal infiltrate of rosacea skin lesions. Using both in vitro and in vivo models, we demonstrate that commensal skin bacteria are necessary for PDC activation and type I IFN production, which, in rosacea, is further amplified by dysbiotic bacteria and antimicrobial peptides with increased type I interferon-inducing capacities. Type I IFNs then drive a pathogenic TH22/TH17 immune response and simultaneously render ECs susceptible to IL22 through upregulation of its receptor. Thereby, type I IFNs link skin dysbiosis to neoangiogenesis, one of the hallmarks of rosacea.

Previous studies have demonstrated that KLK5 and cathelicidin peptides have a functional role in driving an inflammatory response in rosacea. Here, we unravel the underlying pathomechanisms: KLK5, which is overexpressed in rosacea, cleaves cathelicidin into different peptides. The cathelicidin peptides lead to infiltration of the skin by pDCs, which in turn are activated by complexes of these cathelicidin peptides and nucleic acid to produce large amounts of type I IFNs. In a self-amplifying loop, type I IFNs then attract, through induction of CXCR3 ligands, ${ }^{26-28}$ additional pDCs to the skin. The resulting type I IFN overexpression eventually induces the strong TH22/TH17 immune response characteristic for rosacea.

We find that pDCs represent the principal cellular source of IFNa- and - for the most part - of IFN $\beta$ production in vivo. However, some residual IFN $\beta$ and interferon-response gene expression are discernible despite pDC-depletion. Thus, other cell types might contribute to the type I IFN production in rosacea. Keratinocytes ${ }^{29-31}$ and endothelial cells ${ }^{32}$ have indeed been shown to produce IFN $\beta$ in response to sensing nucleic acids complexed with LL-37 via endosomal or cytosolic receptors. 
Self-DNA and self-RNA complexed with antimicrobial peptides are able to activate endosomal TLRs of pDCs. ${ }^{16,17}$ However, we show here that commensal skin bacteria are required as source of nucleic acid in order to induce relevant pDC activation and type I IFN production in vivo, in line with similar observations from skin wound healing. ${ }^{33}$ These findings suggest that bacteria have several key pathogenic roles in rosacea: 1) They activate TLR2 on KCs to upregulate KLK5, 2) provide an essential source for nucleic acid in order to activate pDCs to produce type I IFN and initiate a pathogenic inflammation, and 3) activate neutrophils through cell surface-TLRs. ${ }^{8}$ Therefore, bacteria are essential for the activation of the KLK5 - cathelicidin - pDC/type I IFN axis. On the other hand, via direct activation of neutrophils, they also induce an inflammatory pathway that is independent of pDC activation and type I IFN.

We found that the skin commensal B.oleronius, previously shown to be dysbiotic in rosacea, ${ }^{9}$ further amplifies type I IFN production. In fact, compared to other commensal bacteria, in particular to $C$.acnes, which plays an important pathogenic role in acne - a disease with strong clinical resemblance to rosacea - B. oleronius is highly susceptible and preferentially killed by cathelicidin antimicrobial peptides. This leads to enhanced generation of complexes with DNA facilitating the activation of pDCs. The importance of dysbiotic communities of commensal bacteria in the pathogenesis of rosacea is further supported by the fact that commensal $\mathrm{C}$. acnes shows lower relative abundance in rosacea patients and is further reduced with increased disease severity. ${ }^{34,35}$ On the other hand, $B$. oleronius has been linked to disease activity in rosacea. ${ }^{8,36}$ Demodex mites, which harbour $B$. oleronius, are markedly increased in patients with rosacea as compared to healthy controls. ${ }^{37}$ In fact, Demodex mites are found within the pilosebaceous unit, which is often at the epicentre of the inflammatory infiltrate in papulopustular rosacea. Demodex can directly activate TLR2 and therefore, contribute themselves to the induction of an inflammatory response in rosacea. Nevertheless, papulopustular rosacea improves upon antibiotic treatments, which do not display any activity against Demodex. It has been suggested that the clinical efficacy of tetracyclins is based on their bystander anti-inflammatory properties. However, antiinflammatory treatments, such as topical steroids and tacrolimus often aggravate or even induce rosacea, ${ }^{38,39}$ indicating that antibiotics rather act by alleviating the bacterial burden.

Most studies have shown higher prevalence and incidence of rosacea in women than men and a tendency for earlier diagnosis in women. ${ }^{40}$ The identification of type I IFN as key early driver in rosacea might provide one explanation. Like rosacea, most autoimmune diseases also predominantly affect females. Many immune-associated genes are encoded on the X chromosome. ${ }^{41,42}$ While usually one of the $X$ chromosomes in each female cell should be inactive, approximately $15 \%$ of $X$-linked genes escape inactivation. ${ }^{43}$ Thus, $T L R 7$ and $T L R 8$, due to their localization on the $\mathrm{X}$ chromosome, might be overexpressed in females and could prone them to type I IFN driven inflammation and autoimmunity. This hypothesis is further supported by the finding that in women, rosacea was significantly associated with autoimmune diseases such as type 1 diabetes mellitus, celiac disease, multiple sclerosis, and rheumatoid arthritis. In contrast, only rheumatoid arthritis was associated with rosacea among male patients. ${ }^{44}$ Interestingly, one of the only two genomic regions associated with all these autoimmune 
diseases contains TYK2, ${ }^{45}$ a member of the Janus kinase (JAK) family playing a central role in the type I IFN signalling pathway. ${ }^{46}$ Taken together, sex-biased overexpression of the type I IFN pathway could explain an increased susceptibility for autoimmune diseases and rosacea in women.

While in tumours, type I IFN has anti-angiogenic properties, ${ }^{47}$ we find that in the context of skin inflammation, type I IFN in fact promotes angiogenesis. Yet, type I IFN does not directly affect EC proliferation. Instead, similar to what has been shown for keratinocyte proliferation, ${ }^{48}$ it licences IL22 to induce angiogenesis via upregulation of its receptor on ECs. Thus, type I IFN induces both IL22 at the site of inflammation and concomitant upregulation of the IL22 receptor on ECs, providing the interface between immune activation and angiogenesis. This raises the hypothesis, that repetitive flare-ups with frequent type I IFN bursts and IL22R upregulation on ECs leads to pronounced neoangiogenesis and eventually to permanent erythema, an observation that is well known from the clinic.

We have found that the different cathelicidins peptides present in rosacea show differential abilities to kill various bacterial strains and to activate the immune system. Cathelicidins, which belong to the ancestral and evolutionary conserved AMPs, are critical in the innate defence against invasion of pathogenic microbes ${ }^{49}$ and deficiency leads to increased rates of infection. Moreover, overexpression of cathelicidins and other AMPs is critically implicated in driving inflammatory and autoimmune diseases. ${ }^{50,51}$. Here, we show that antimicrobial potency of cathelicidin peptides and their ability to activate the immune system are clearly distinct. The differential ability or lack thereof to induce type I IFN was dependent on their respective capacity to bind and condense DNA, to internalize nucleic acid and thereby enable them to activate TLR7 and TLR9 in pDCs. At least partially, this seems to be linked to the different charges of these peptides. The alpha helical structure of cathelicidin peptides, which stabilizes interactions between the cationic peptide and the DNA helix, ${ }^{52}$ as well as the sequence and conformation of LL-37 and FR-29 are similar. However, FR-29 (+8) has a higher net-positive charge than LL-37 (+6) and thus, seems to provide a more optimal peptide-DNA charge ratio in order to activate pDCs. ${ }^{53}$ The question remains, why - from an evolutionary viewpoint - various cathelicidin peptides should be present in the skin. Plenty of different cathelicidin peptides with diverging antimicrobial capacities might broaden the spectrum and improve defence against invading pathogens. However, there seems to be a fine line between control of the microbiota and the instigation of an overt immune response by commensal bacteria as seen in rosacea.

A pathogenic role for the induction of inflammation and immune responses by pDC-derived type I IFN has been shown for several diseases and drug side effects such as psoriasis, lupus, and anti-TNF-induced paradoxical psoriasis. ${ }^{21,28,54}$ In fact, a genetically determined dysregulation of the pDC-type I IFN pathway has been shown for several autoimmune diseases including psoriasis and lupus. Positive familial history in patients, higher incidence rates of rosacea in Celtic and Northern European countries, and identification of risk gene variants ${ }^{55}$ suggest a genetic predilection also for rosacea. It remains to be elucidated if such susceptibility genes can be linked to the type I IFN pathway in rosacea. 
The common underlying pathway raises the question how an early type I IFN overproduction by pDCs can lead to the great diversity among these entities and initiate such different clinical phenotypes and distinct immune responses. Psoriasis is a T cell mediated disease, lupus is B cell-mediated, and paradoxical psoriasis represents an ongoing innate inflammation. ${ }^{56}$ One possibility is that the genetic background of the patient defines the immune response and clinical phenotype induced by type I IFN. This hypothesis is supported by the clinical observation that type I IFN therapy of diseases such as hepatitis C, lymphoma, multiple sclerosis, or melanoma, can lead to a vast spectrum of different immune mediated side effects and autoimmune adverse events. ${ }^{57,58}$ Then again, additional factors probably contribute to the unique phenotype of rosacea and its limitation to the central facial area: the commensal bacteria initiating the immune response in rosacea are associated with Demodex mites, which themselves are restricted to sebaceous area of the face. The face is particularly exposed to UV irradiation and temperature changes, known trigger factors for rosacea flare-ups. In addition, a neurovascular dysregulation contributes to the characteristic facial flushing in rosacea. ${ }^{59,60}$

While both anti-IFNa ${ }^{61}$ and anti-IL $22^{62}$ failed to meet primary endpoints in clinical trials for chronic plaque psoriasis, immunotherapies targeting $\mathrm{pDCs}^{63}$ and the type I IFN pathway ${ }^{64}$ have recently demonstrated promising results for the treatment of lupus. Moreover, IL-22 has been linked to severity of skin disease in lupus patients ${ }^{65}$ and blockade of upstream IL-12/23 has shown clinical efficacy in patients with systemic lupus. ${ }^{66}$

Taken together, this study directly links dysbiosis to the induction of pathogenic skin inflammation and neoangiogenesis. Furthermore, our findings might provide basis for the design of new therapeutic strategies targeting bacteria subpopulations, pDCs and type I IFN, or IL22 for the treatment of rosacea and prevention of acute flare-ups.

\section{Materials And Methods}

Human samples. Studies were approved by the local institutional review board of Lausanne, Switzerland (ethical approval number 265/12) and Dusseldorf, Germany (ethical approval number 2048). After dermatopathological assessment, punch-biopsies were taken with informed consent from affected skin (cheeks, nose, chin, or forehead) from acute flare-ups or stabilised rosacea lesions. Samples were selected where a Cutaneous Lupus Erythematous diagnosis was unequivocally ruled out by immunopathological assessment. In total, 16 biopsies were obtained from 16 patients during acute flares, and 16 biopsies from 16 different patients during stable disease (Suppl. Table 1). Biopsies from healthy individuals were obtained with informed consent from residual skin from aesthetic surgery of healthy individuals. Buffy coats from healthy donors were obtained from the local transfusion centre and blood bank, with ethical approval from cantonal authorities.

Mouse experimentation. Animal experiments were performed according to institutional and the Swiss Federal Animal Protection Act and cantonal laws on animal protection. Consent was received from the Swiss Federal Food Safety and Veterinary Office. Balb/cByJ (JAX mouse strain) mice were purchased 
from Charles River Laboratories France and experiments performed on age-matched animals. Intradermal injections of cathelicidin peptides were performed as previously described unless stated otherwise. Briefly, a $50 \mu \mathrm{L}$ volume of sterile saline, or $250 \mu \mathrm{M}$ of synthetic cathelicidin peptides (custom made from Proteogenix, France) was injected intradermally 4 times every $12 \mathrm{~h}$ and mice euthanized and biopsied $48 \mathrm{~h}$ after first injection. Antibodies anti-Ifnar1 (MAR1-5A3, BioXCell) and pDC depleting antibodies (BX444, BioXCell) were each injected $(200 \mu \mathrm{g}) 24 \mathrm{~h}$ prior to intradermal cathelicidin injections. pDC depletion in Balb/c BDCA2-DTR transgenic mice was performed by injection of 120ng of diphtheria toxin 24h and $4 \mathrm{~h}$ prior to the first intradermal LL-37 injection. Topical antibiotic treatments were performed for $48 \mathrm{~h}$ prior to intradermal injections in two applications, with a tailor made ointment preparation (Vaselinum album qsp $30 \mathrm{~g}$, paraffinum liquidum $5 \mathrm{~g}$ ) either containing or lacking Neomycin (105mg), Polymyxin B (18.85mg), and Bacitracinum (141.17mg), purchased from International Pharmacy, Golaz Chemist SA. For B.oleronius (ATCC ${ }^{8} 700005^{\mathrm{TM}}$ ) injections, the corresponding amount of bacterium inoculate for $10^{4} \mathrm{CFU}$ after overnight culture was heat-killed at $55^{\circ} \mathrm{C}$ for 10 minutes (killing confirmed by overnight culture) and either incubated with $250 \mu \mathrm{M}$ of LL-37, or vehicle, for $12 \mathrm{~h}$, and intradermal injections performed at $12 \mathrm{~h}$ intervals.

Immunohistochemistry. For immunohistochemistry of human skin, samples were fixed in $4 \%$ paraformaldehyde and paraffin-embedded. Stainings were performed using anti-CD123 (7G3, BD Pharmingen) followed by visualisation using the horseradish peroxidase technique. The number of positive cells per high magnification field determined in relation to the number non-stromal inflammatory cells using automated and standardized counting of round nuclei (ImageJ v1.50b).

Immunofluorescence. Four-mm skin biopsies from rosacea patients were snap-frozen in liquid nitrogen and embedded in OCT compound prior to frozen sectioning on a microtome-cryostat. Then, $10-\mu \mathrm{m}$ skin sections were fixed with cold methanol for $5 \mathrm{~min}$ at $-20^{\circ} \mathrm{C}$, permeabilized in PBS $0.012 \%$ Triton X-100 for 20 seconds at RT, and saturated with PBS 5\% normal fetal bovine serum for 30 min at RT. Skin sections were then stained in Dako REAL Antibody Diluent (Dako) with the following fluorophore-conjugated monoclonal antibodies: BDCA-2-FITC (1/50, Miltenyi, clone AC144), CD11C-PE (1/5, BD Biosciences, clone S-HCL-3), and CD123-APC (1/20, BioLegend, clone 6H6), for 2 hours at RT. Following several washes, slides were mounted with ProLong Gold antifade mountant with DAPI (Thermo Fisher Scientific) and analyzed on a Zeiss LSM 700 confocal microscope.

Gene expression analyses. For quantitative real-time PCR, samples were homogenized by mechanical disruption using Polytron PT1200E (Kinematica) in Trizol reagent (Invitrogen) and total RNA obtained using phenol/chloroform extraction, and isopropanol followed by ethanol precipitation. RNA was reverse transcribed using SuperScript II reverse transcriptase kit (\#18064014, Invitrogen), using random primers (C1181, promega) and Oligo (dT)15 primers (C100A, promega). Quantitative PCR was performed using "Best Coverage" TaqMan ${ }^{\circledR}$ probes or SybrGreen ${ }^{\circledR}$ primers (sequences indicated in Supplementary Table 2). TaqMan ${ }^{\circledR}$ Gene Expression Mix (Lifetechnologies) or Power SybrGreen ${ }^{\circledR}$ Master Mix were used for qPCR in $10 \mu \mathrm{L}$ reaction volumes. Values are expressed as $2 \Delta-\Delta \mathrm{Ct} \times 104$ relative to the endogenous control GAPDH/Gapdh and for human samples these were normalised to healthy donor expression. 
Flow cytometry. Biopsies of $0.6 \mathrm{~cm}$ in diameter were taken of the injection site, and skin was mechanically disrupted using a sterile scalpel in PBS containing 2mM EDTA. The resulting cell suspension was stained in 0.5\% FBS/PBS 2mM EDTA using anti-B220 (RA3-6B2, BD Pharmingen), anti-Cd11c (N418, eBiosciences), anti-Cd45 (30-F11, BD Pharmingen), anti-Pdca1 (927, Biolegend), anti-Cd31 (390, eBiosciences), anti-Ki67 (SolA15, eBiosciences), anti-BrdU (Bu20A, eBiosciences).

Structure prediction of AMPs. Peptide prediction was performed using the I-TASSER tool ${ }^{67,68}$ and visualised using Chimera 1.14. ${ }^{69}$

pDC stimulations. pDCs were isolated from Buffy Coats from healthy donours using the Diamond Plasmacytoid Dendritic Cell Isolation Kit II (130-097-415, Miltenyi), and cultured 5x104 cells/200 $\mu \mathrm{L}$ in RPMI 1640 supplemented with $10 \%$ FBS and 1\% penicillin/streptomycin. Stimulations were performed using $5 \mu \mathrm{g} / \mathrm{mL}$ human DNA and $10 \mu \mathrm{M}$ or $3 \mu \mathrm{M}$ of the indicated peptides. Live B.oleronius was added at $105 \mathrm{CFU} / \mathrm{mL}$ alone or in combination with $5 \mu \mathrm{M}$ of LL-37. IFNa production was measured by ELISA after $24 \mathrm{~h}$ of stimulation using the human pan IFN-a specific development kit (3425-1H-6, Mabtech).

DNA-cathelicidin peptide complex uptake. Isolated pDCs were incubated with $3 \mu \mathrm{g} / \mathrm{mL}$ of Alexa Fluor 488labeled DNA (U21650, Invitrogen) alone or in combination with $1 \mu \mathrm{M}$ of the indicated peptide at $37^{\circ} \mathrm{C}$ for 3h in RPMI 1640 (Gibco) supplemented with 10\% FBS.

DNA binding. DNA binding and condensation activity of the cathelicidin peptides was determined using a picogreen (Invitrogen) dye fluorescence quenching technique. Peptides were added to 2 ug of purified human genomic DNA at the indicated final concentrations for 30 minutes, and picogreen dye (Quant-iT ${ }^{\mathrm{TM}}$ PicoGreen ${ }^{\text {TM }}$ dsDNA Assay, Invitrogen) used for DNA staining. Sample fluorescence was determined using $480 \mathrm{~nm}$ excitation and measured at $520 \mathrm{~nm}$ with continuous reading between [500-650nm] using a spectrofluorometer. When there is strong peptide-DNA binding and condensation, condensed DNA molecules are rendered inaccessible to the dye leading to reduction in signal intensity.

In vitro and in vivo Cramp processing. Mouse Cramp protein (RPC419Mu01, Cloud-Clone Corp.) 100ng was incubated with 1.5ng hKLK5 (1108-SE, R\&D Systems) or reconstitution buffer for the indicated times, heated to $95 \mathbb{C}$ for 5 minutes, and ran by western blot alongside synthetic mCramp petide (Proteogenix, France). Same size skin punch biopsies from 7-day old K5.KLK5 mice and wild-type littermates was homogenised using Polytron PT-1200E (Kinematica) in the presence of protease inhibitor cocktail (P8340, Sigma), and immediately after SDS was added to a $0.4 \%$ final concentration, samples were sonicated before being heated to $95 \mathbb{C}$ for 5 minutes, and ran by western blot alongside mCramp peptide and protein. Actin was probed as loading control.

Bacterial killing. Bacterial strains Bacillus oleronius (ATCC $\AA 700005^{\mathrm{TM}}$ ), Staphylococcus epidermidis (ATCC $\AA 14990^{\mathrm{TM}}$ ), Cutibacterium acnes (ATCC $\AA 6919^{\mathrm{TM}}$ ), Klebsiella pneumoniae (ATCC $\AA 10031^{\mathrm{TM}}$ ), Pseudomonas aeruginosa (ATCC $\AA 27853^{\mathrm{TM}}$ ) Escherichia coli (ATCC $\AA 700973^{\mathrm{TM}}$ ) were prepared at the indicated working concentrations and incubated with the indicated peptide at working concentrations 
ranging $0.2-10 \mu \mathrm{M}$ for $5 \mathrm{~h}$ in PBS, plated and CFU were counted after overnight incubation at $37^{\circ} \mathrm{C}$, except for $C$.acnes which was incubated under anaerobic conditions. Values are expressed as percentage of survival.

Huvec culture, proliferation, and survival. Human umbilical vein endothelial cells (ATCC® CRL-1730 ${ }^{\mathrm{TM}}$ ) were cultured in growth media (EGM ${ }^{\mathrm{TM}}$-2 Endothelial Cell Growth Medium-2 BulletKit ${ }^{\mathrm{TM}}$, Lonza) containing growth factors IGF1, FGF2, EGF, VEGFA according to the manufacturer's instructions, unless otherwise stated. Cells ( $5 \times 10^{3}$ per 96-well) were stimulated with $1000 \mathrm{U} / \mathrm{mL}$ human IFNa (1100-1, PBL), and/or 100ng/mL IL22 (200-22, PeproTech) for 8h for gene expression analyses, or 5 days for survival and proliferation analyses. For survival experiments cells were stained with SYTO®13/SytoX® ORANGE (S34854, S11368, Invitrogen) and analysed by flow cytometry. Live cells were counted as total Syto single-positive cells per condition. For proliferation experiments, cells were fixed, permeabilised (FIX \& PERM Cell Fixation \& Permeabilization Kit, ThermoFisher), stained for KI67, and analysed by flow cytometry.

Mouse endothelial cell proliferation. To determine in vivo mouse endothelial cell proliferation, mice were treated with the indicated antibody prior to induction of cathelicidin mediated skin inflammation as previously, and injected intraperitoneally with $2.5 \mathrm{mg}$ of BrdU (19-160, Merck) in $200 \mu \mathrm{L}$ saline $24 \mathrm{~h}$ prior to end of the injection regimen. Biopsies measuring $0.28 \mathrm{~cm}^{2}$ of the injection sites were taken, cell suspensions were prepared as described previously, stained for $\mathrm{Cd} 45$ and $\mathrm{Cd} 31$, fixed and permeabilised as described in the previous section, and stained for Ki67 and BrdU. The percentage of proliferating cells was determined as single-positive Ki67 (initiating proliferation), Ki67-BrdU double positive cells (sustained proliferation), and single-positive BrdU (previously proliferating).

Videocapillaroscopy and human dermoscopy. For mouse skin videocapillaroscopy, mice were anaesthetised with a ketamine-xylazine cocktail $(100 \mathrm{mg} / \mathrm{kg}$ and $10 \mathrm{mg} / \mathrm{kg})$ to reduce mobility and slow respiration for more stable imaging. Injection sites and adjacent areas were delicately ointed with an immersion oil, and microvessels visualised using a clinical nailfold videocapillaroscope Xport Technology capXview, images were acquired with the software capXview HD2 version 5.0. Microvessel numbers were determined by manual counting from images of injection sites and adjacent areas using ImageJ v.1.8.0_191. Rosacea microvessels were visualised with a FotoFinder ${ }^{\circledR}$ medicam 800 HD.

\section{References}

1. Gether, L., Overgaard, L. K., Egeberg, A. \& Thyssen, J. P. Incidence and prevalence of rosacea: a systematic review and meta-analysis. Br. J. Dermatol. 179, 282-289 (2018).

2. Haber, R. \& El Gemayel, M. Comorbidities in rosacea: A systematic review and update. Journal of the American Academy of Dermatology (2018) doi:10.1016/j.jaad.2017.09.016.

3. Moustafa, F., Lewallen, R. S. \& Feldman, S. R. The psychological impact of rosacea and the influence of current management options. Journal of the American Academy of Dermatology (2014) 
doi:10.1016/j.jaad.2014.05.036.

4. Forton, F. \& Seys, B. Density of Demodex folliculorum in rosacea: A case-control study using standardized skin-surface biopsy. Br. J. Dermatol. (1993) doi:10.1111/j.1365-2133.1993.tb00261.x.

5. Erbağci, Z. \& Özgöztaşi, O. The significance of Demodex folliculorum density in rosacea. Int. J. Dermatol. 37, 421-425 (1998).

6. Bonnar, E., Eustace, P. \& Powell, F. C. The Demodex mite population in rosacea. J. Am. Acad. Dermatol. 28, 443-448 (1993).

7. Jarmuda, S. et al. Correlation between serum reactivity to Demodex-associated Bacillus oleronius proteins, and altered sebum levels and Demodex populations in erythematotelangiectatic rosacea patients. J. Med. Microbiol. 63, 258-262 (2014).

8. O’Reilly, N., Bergin, D., Reeves, E. P., McElvaney, N. G. \& Kavanagh, K. Demodex-associated bacterial proteins induce neutrophil activation. Br. J. Dermatol. 166, 753-760 (2012).

9. Lacey, N., Delaney, S., Kavanagh, K. \& Powell, F. C. Mite-related bacterial antigens stimulate inflammatory cells in rosacea. Br. J. Dermatol. 157, 474-481 (2007).

10. Yamasaki, K. et al. TLR2 expression is increased in rosacea and stimulates enhanced serine protease production by keratinocytes. J. Invest. Dermatol. 131, 688-697 (2011).

11. Schauber, J. et al. Injury enhances TLR2 function and antimicrobial peptide expression through a vitamin D-dependent mechanism. J. Clin. Invest. 117, 803-811 (2007).

12. Yamasaki, K. et al. Kallikrein-mediated proteolysis regulates the antimicrobial effects of cathelicidins in skin. Faseb J 20, 2068-2080 (2006).

13. Yamasaki, K. et al. Increased serine protease activity and cathelicidin promotes skin inflammation in rosacea. Nat. Med. 13, 975-980 (2007).

14. Koczulla, R. et al. An angiogenic role for the human peptide antibiotic LL-37/hCAP-18. J. Clin. Invest. 111, 1665-1672 (2003).

15. Tjabringa, G. S., Ninaber, D. K., Drijfhout, J. W., Rabe, K. F. \& Hiemstra, P. S. Human cathelicidin LL-37 Is a chemoattractant for eosinophils and neutrophils that acts via formyl-peptide receptors. Int. Arch. Allergy Immunol. 140, 103-112 (2006).

16. Lande, R. et al. Plasmacytoid dendritic cells sense self-DNA coupled with antimicrobial peptide. Nature 449, 564-569 (2007).

17. Ganguly, D. et al. Self-RNA-antimicrobial peptide complexes activate human dendritic cells through TLR7 and TLR8. J. Exp. Med. 206, 1983-1994 (2009).

18. Gregorio, J. et al. Plasmacytoid dendritic cells sense skin injury and promote wound healing through type I interferons. J. Exp. Med. 207, 2921-2930 (2010).

19. Conrad, C., Meller, S. \& Gilliet, M. Plasmacytoid dendritic cells in the skin: to sense or not to sense nucleic acids. Semin Immunol 21, 101-109 (2009).

20. Gilliet, M. et al. Psoriasis triggered by toll-like receptor 7 agonist imiquimod in the presence of dermal plasmacytoid dendritic cell precursors. Arch. Dermatol. (2004) doi:10.1001/archderm.140.12.1490. 
21. Nestle, F. O. et al. Plasmacytoid predendritic cells initiate psoriasis through interferon- production. J. Exp. Med. 202, 135-143 (2005).

22. Buhl, T. et al. Molecular and Morphological Characterization of Inflammatory Infiltrate in Rosacea Reveals Activation of Th1/Th17 Pathways. J. Invest. Dermatol. 135, 2198-208 (2015).

23. Furio, L. et al. Transgenic kallikrein 5 mice reproduce major cutaneous and systemic hallmarks of Netherton syndrome. J. Exp. Med. 211, 499 LP - 513 (2014).

24. Lande, R. et al. Neutrophils Activate Plasmacytoid Dendritic Cells by Releasing Self-DNA-Peptide Complexes in Systemic Lupus Erythematosus. Sci. Transl. Med. 3, 1-11 (2011).

25. Zhang, Z. et al. Mitochondrial DNA-LL-37 Complex Promotes Atherosclerosis by Escaping from Autophagic Recognition. Immunity 43, 1137-1147 (2015).

26. Vanbervliet, B. et al. The inducible CXCR3 ligands control plasmacytoid dendritic cell responsiveness to the constitutive chemokine stromal cell-derived factor 1 (SDF-1)/CXCL12. J. Exp. Med. 198, 823830 (2003).

27. Meller, S. et al. Ultraviolet radiation-induced injury, chemokines, and leukocyte recruitment: An amplification cycle triggering cutaneous lupus erythematosus. Arthritis Rheum. (2005) doi:10.1002/art.21034.

28. Conrad, C. et al. TNF blockade induces a dysregulated type I interferon response without autoimmunity in paradoxical psoriasis. Nat. Commun. 9, (2018).

29. Zhang, L. J. et al. Antimicrobial Peptide LL37 and MAVS Signaling Drive Interferon- $\beta$ Production by Epidermal Keratinocytes during Skin Injury. Immunity 45, 119-130 (2016).

30. Takiguchi, T. et al. Cathelicidin antimicrobial peptide LL-37 augments interferon- $\beta$ expression and antiviral activity induced by double-stranded RNA in keratinocytes. Br. J. Dermatol. 171, 492-498 (2014).

31. Takahashi, T. et al. Cathelicidin promotes inflammation by enabling binding of self-RNA to cell surface scavenger receptors. Sci. Rep. 8, 1-13 (2018).

32. Kulkarni, N. N. et al. Innate Immune Dysfunction in Rosacea Promotes Photosensitivity and Vascular Adhesion Molecule Expression. J. Invest. Dermatol. 140, 645-655.e6 (2020).

33. Di Domizio, J. et al. The commensal skin microbiota triggers type I IFN-dependent innate repair responses in injured skin. Nat. Immunol. 139, S275 (2020).

34. Woo, Y. R., Lee, S. H., Cho, S. H., Lee, J. D. \& Kim, H. S. Characterization and Analysis of the Skin Microbiota in Rosacea: Impact of Systemic Antibiotics. J. Clin. Med. 9, 185 (2020).

35. Rainer, B. M. et al. Characterization and Analysis of the Skin Microbiota in Rosacea: A Case-Control Study. Am. J. Clin. Dermatol. 21, 139-147 (2020).

36. Li, J. et al. Correlation between Ocular Demodex Infestation and Serum Immunoreactivity to Bacillus Proteins in Patients with Facial Rosacea. Ophthalmology (2010) doi:10.1016/j.ophtha.2009.09.057.

37. Forton, F. M. N. \& De Maertelaer, V. Two consecutive standardized skin surface biopsies: An improved sampling method to evaluate demodex density as a diagnostic tool for rosacea and demodicosis. 
Acta Derm. Venereol. 97, 242-248 (2017).

38. Antille, C., Tran, C., Sorg, O. \& Saurat, J. H. Penetration and metabolism of topical retinoids in ex vivo organ-cultured full-thickness human skin explants. Skin Pharmacol. Physiol. 17, 124-128 (2004).

39. Jarmuda, S. et al. Potential role of Demodex mites and bacteria in the induction of rosacea. J. Med. Microbiol. 61, 1504-1510 (2012).

40. Spoendlin, J., Voegel, J. J., Jick, S. S. \& Meier, C. R. A study on the epidemiology of rosacea in the U.K. Br. J. Dermatol. 167, 598-605 (2012).

41. Rubtsova, K., Marrack, P. \& Rubtsov, A. V. TLR7, IFNc, and T-bet: Their roles in the development of ABCs in femalebiased autoimmunity. Cell. Immunol. (2015) doi:10.1016/j.cellimm.2014.12.002.

42. Libert, C., Dejager, L. \& Pinheiro, I. The $X$ chromosome in immune functions: When a chromosome makes the difference. Nat. Rev. Immunol. 10, 594-604 (2010).

43. Carrel, L. \& Willard, H. F. X-inactivation profile reveals extensive variability in X-linked gene expression in females. Nature 434, 400-404 (2005).

44. Egeberg, A., Hansen, P. R., Gislason, G. H. \& Thyssen, J. P. Clustering of autoimmune diseases in patients with rosacea. J. Am. Acad. Dermatol. 74, 667-672.e2 (2016).

45. Fortune, M. D. et al. Statistical colocalization of genetic risk variants for related autoimmune diseases in the context of common controls. Nat. Genet. 47, 839-846 (2015).

46. Prchal-Murphy, M. et al. TYK2 kinase activity is required for functional type I interferon responses in Vivo. PLoS One 7, 1-12 (2012).

47. Parker, B. S., Rautela, J. \& Hertzog, P. J. Antitumour actions of interferons: Implications for cancer therapy. Nat. Rev. Cancer 16, 131-144 (2016).

48. Tohyama, M. et al. IFN-a Enhances IL-22 Receptor Expression in Keratinocytes: A Possible Role in the Development of Psoriasis. J. Invest. Dermatol. 132, 1933-1935 (2012).

49. Kościuczuk, E. M. et al. Cathelicidins: family of antimicrobial peptides. A review. Mol. Biol. Rep. 39, 10957-10970 (2012).

50. Kahlenberg, J. M. \& Kaplan, M. J. Little Peptide, Big Effects: The Role of LL-37 in Inflammation and Autoimmune Disease. J. Immunol. 191, 4895-4901 (2013).

51. Gilliet, M. \& Lande, R. Antimicrobial peptides and self-DNA in autoimmune skin inflammation. Curr. Opin. Immunol. 20, 401-407 (2008).

52. Niidome, T., Wakamatsu, M., Wada, A., Hirayama, T. \& Aoyagi, H. Required structure of cationic peptide for oligonucleotide-binding and -delivering into cells. J. Pept. Sci. 6, 271-279 (2000).

53. Lande, R. et al. Cationic antimicrobial peptides in psoriatic skin cooperate to break innate tolerance to self-DNA. Eur. J. Immunol. 45, 203-213 (2015).

54. Garcia-Romo, G. S. et al. Netting neutrophils are major inducers of type I IFN production in pediatric systemic lupus erythematosus. Sci. Transl. Med. 3, (2011).

55. Chang, A. L. S. et al. Assessment of the Genetic Basis of Rosacea by Genome-Wide Association Study. 2 J. Investig. Dermatology 00, 1-8 (2015). 
56. Mylonas, A. \& Conrad, C. Psoriasis: Classical vs. Paradoxical. The Yin-Yang of TNF and Type I Interferon. Front. Immunol. 9, (2018).

57. Dusheiko, G. Side effects of a interferon in chronic hepatitis C. Hepatology 26, 112S-121S (1997).

58. Sleijfer, S., Bannink, M., Van Gool, A. R., Kruit, W. H. J. \& Stoter, G. Side effects of interferon-a therapy. Pharm. World Sci. 27, 423-431 (2005).

59. Schwab, V. D. et al. Neurovascular and Neuroimmune Aspects in the Pathophysiology of Rosacea. J. Investig. Dermatology Symp. Proc. 15, 53-62 (2011).

60. Steinhoff, M., Schmelz, M. \& Schauber, J. Facial erythema of rosacea - Aetiology, different pathophysiologies and treatment options. Acta Derm. Venereol. 96, 579-589 (2016).

61. Bissonnette, R. et al. A randomized, double-blind, placebo-controlled, phase I study of MEDI-545, an anti-interferon-alfa monoclonal antibody, in subjects with chronic psoriasis. J. Am. Acad. Dermatol. 62, 427-436 (2010).

62. NCT01010542 \& Pfizer. Study Evaluating Single Dose Of ILV-095 In Psoriasis Subjects. https://clinicaltrials.gov/ct2/show/NCT01010542.

63. Furie, R. et al. Monoclonal antibody targeting BDCA2 ameliorates skin lesions in systemic lupus erythematosus. J. Clin. Invest. 129, 1359-1371 (2019).

64. Morand, E. F. et al. Trial of anifrolumab in active systemic lupus erythematosus. N. Engl. J. Med. 382, 211-221 (2020).

65. Zhong, W. et al. Elevated levels of CCR6+ T helper 22 cells correlate with skin and renal impairment in systemic lupus erythematosus. Sci. Rep. 7, 1-11 (2017).

66. van Vollenhoven, R. F. et al. Efficacy and safety of ustekinumab, an IL-12 and IL-23 inhibitor, in patients with active systemic lupus erythematosus: results of a multicentre, double-blind, phase 2, randomised, controlled study. Lancet 392, 1330-1339 (2018).

67. Roy, A., Kucukural, A. \& Zhang, Y. I-TASSER: A unified platform for automated protein structure and function prediction. Nat. Protoc. 5, 725-738 (2010).

68. Yang, J. et al. The I-TASSER suite: Protein structure and function prediction. Nat. Methods 12, 7-8 (2014).

69. Pettersen, E. F. et al. UCSF Chimera - A visualization system for exploratory research and analysis. J. Comput. Chem. 25, 1605-1612 (2004).

\section{Declarations}

Acknowledgments: We appreciate excellent technical assistance of Isabelle Surbeck and Ana Joncic at the department of dermatology. We thank Dr. Stefanie Hendrikx and Dr. Borja Luri Prat for invaluable discussions and advice on endothelial cells and the process of angiogenesis. We thank technical assistance by chief nurse Sarah Grognuz, at the department of angiology. We sincerely thank all patients involved in the study. 
Funding: Swiss National Science Foundation (FN 310030-156173) to C.C. Grant from Spirig Pharma SA Foundation to A.M. Grant from Gottfried\&Julia Bangerter-Rhyner foundation to C.C. and A.M.

Author contributions: A.M. and C.C. formulated the hypotheses, designed and supervised the study and experiments, interpreted data, and wrote the manuscript. A.M. performed experiments. O.D. provided critical expertise on the in vivo model. H.C.H, S.M., and B.H. provided human rosacea biopsies and performed expression profiling of samples, and contributed critical appraisal of the manuscript. O.D., J.D.D. and M.G. provided invaluable appraisal of the manuscript, and shaped the hypothesis of the work. Y.W. and A.H. provided transgenic mouse models, samples, shaped the hypothesis of the work, and contributed critical appraisal of the manuscript. L.M. provided invaluable expertise on videocapillaroscopy and angiogenesis, and contributed critical appraisal of the manuscript.

Competing interests: The authors declare no conflict of interest.

\section{Figures}



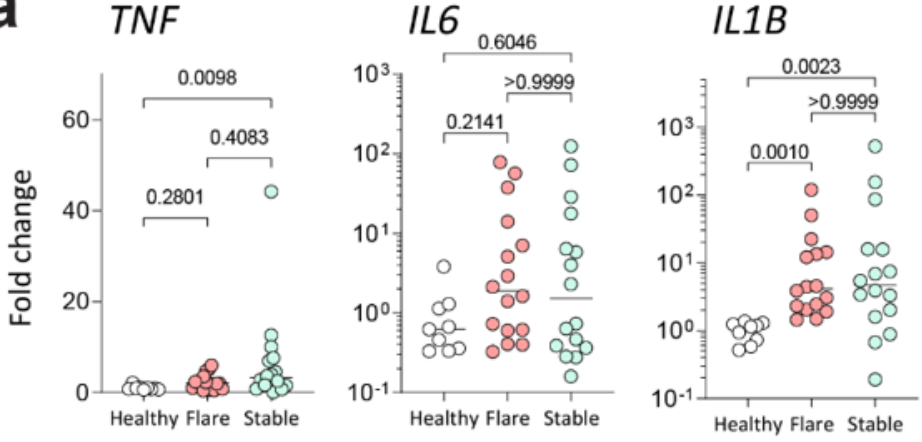

IL10

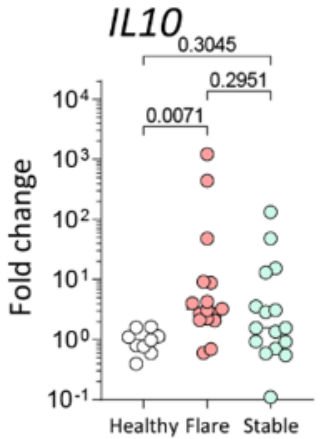

b

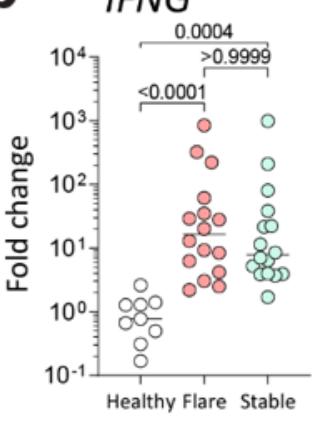

IL12B

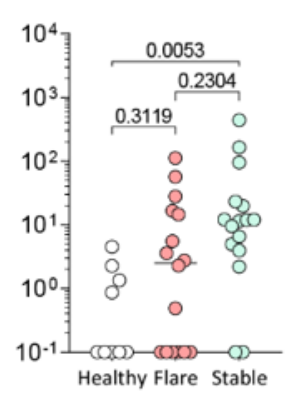

IL17A

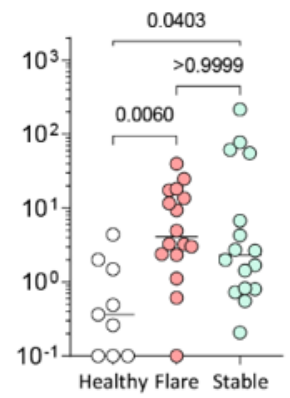

IL23A

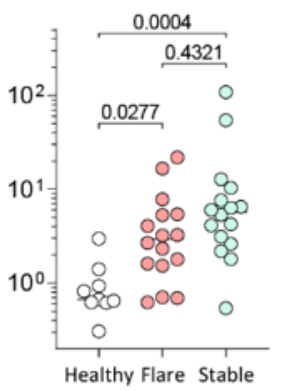

IL-17F

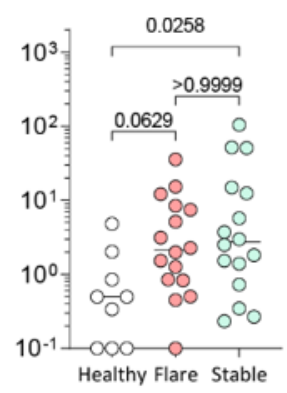

IL8

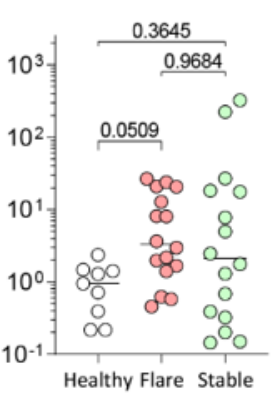

IFNA2

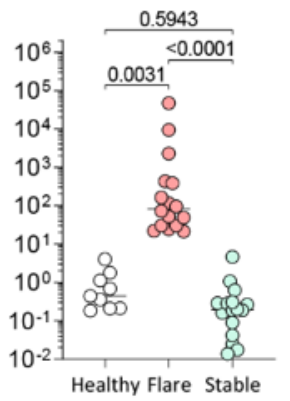

IL-36B

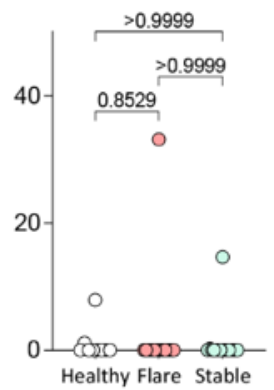

IL22

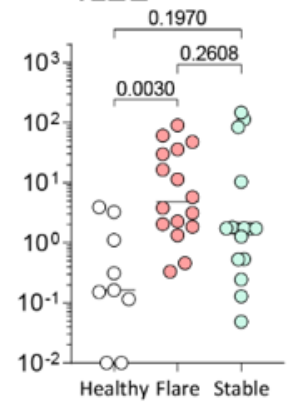

IFNB1

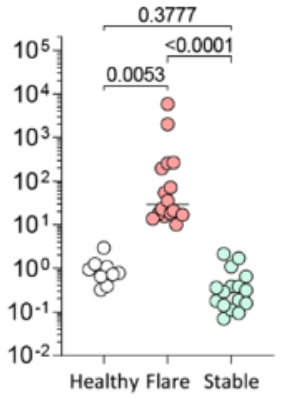

C $M X 1$

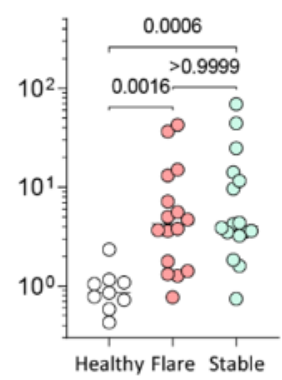

IL13

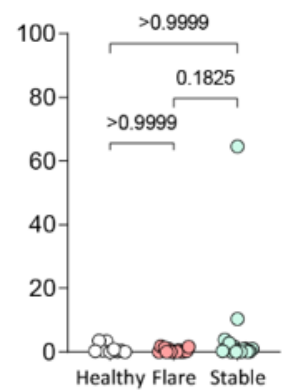

\section{Figure 1}

Inflammatory cytokine profile in acute flare-ups and stabilised lesions of rosacea. (a) Gene expression of innate and (b) adaptive inflammatory genes from acute flare-ups ( $r e d, n=16)$ and stabilised lesions (green, $n=16$ ) as compared to healthy skin (white, $n=9$ ). (c) MX1 expression comparison between healthy skin, flare-ups and stabilised lesions. Multiplicity adjusted P-values of one-way ANOVA with Dunn's multiple comparisons test are depicted. 


\section{a}

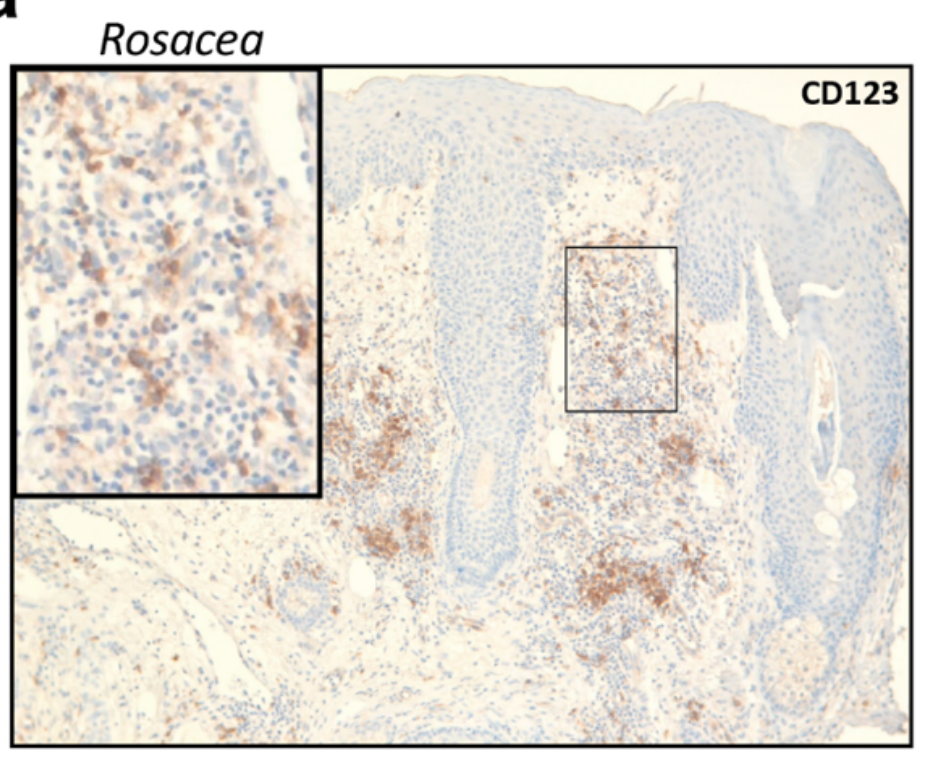

Healthy
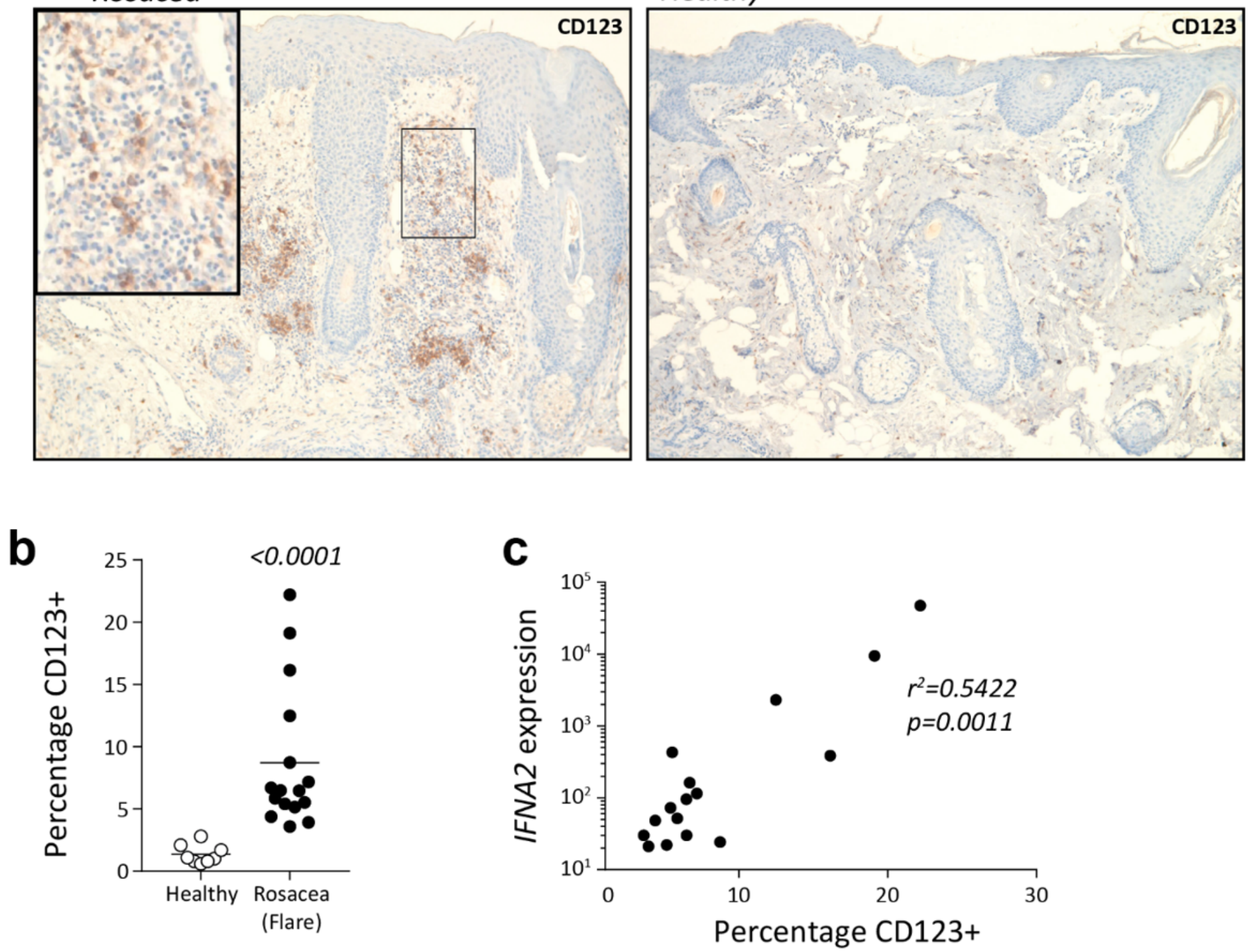

\section{Figure 2}

Type I interferon expression correlates with pDC infiltration in acute flares of rosacea. (a) Immunofluorescence microscopy of rosacea samples co-stained for BDCA2 (green), CD123 (yellow), CD11c (magenta), and DAPI (blue). (b) Rosacea flare-up and healthy skin sections stained for CD123+ pDCs. (c) Percentages of CD123+ pDCs were determined in relation to non-stromal inflammatory cells per high magnification field as mean of triplicate measurements per patient sample. (d) Percentage CD123+ pDCs pictured in (c) plotted against IFNA2 expression from lesions during flare-ups pictured in (Fig. 1a). Non-linear regression with least-squares fit is depicted, with corresponding $\mathrm{p}$ - and $\mathrm{r} 2$ values. 

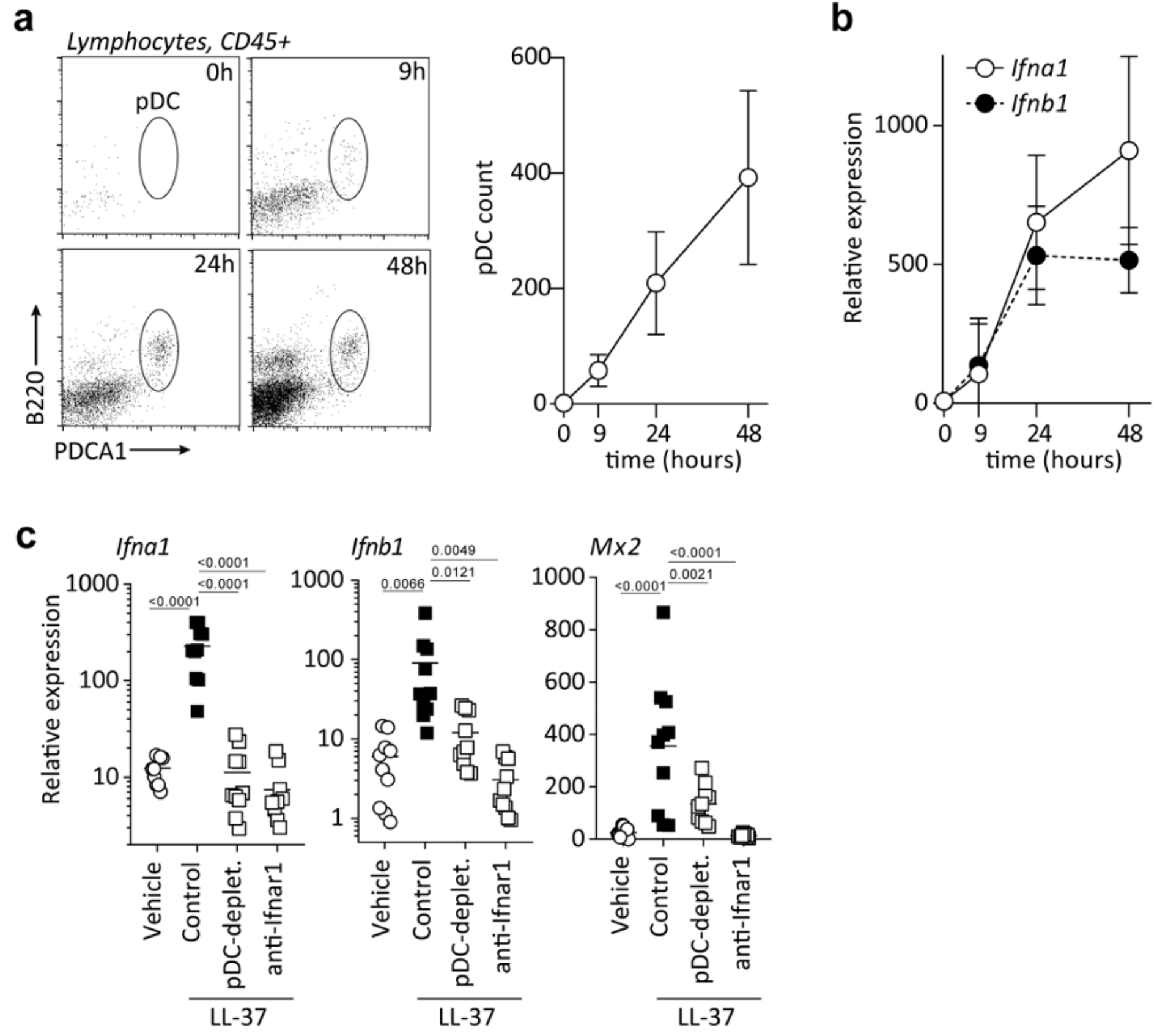

\section{Figure 3}

Type I interferon and downstream response gene expression require pDCs in vivo. (a) Dotplots from mouse skin biopsies of LL-37 intradermal injections, pre-gated on CD45+ lymphocytes at the indicated timepoints and quantification of pDC infiltration, and (b) Ifna1 and Ifnb1 expression. Values expressed as means \pm SD of pooled data from three independent experiments. (c) Gene expression from biopsies following LL-37 intradermal injection in mice depleted of pDCs or blockaded of type I IFN signalling. Results depicted of two pooled experiments. (d) Dotplots and quantification from skin biopsies of LL-37 intradermally injected mice, with or without prior antibody-mediated Ifnar blockade. Multiplicity adjusted P-values of one-way ANOVA are depicted. 
a
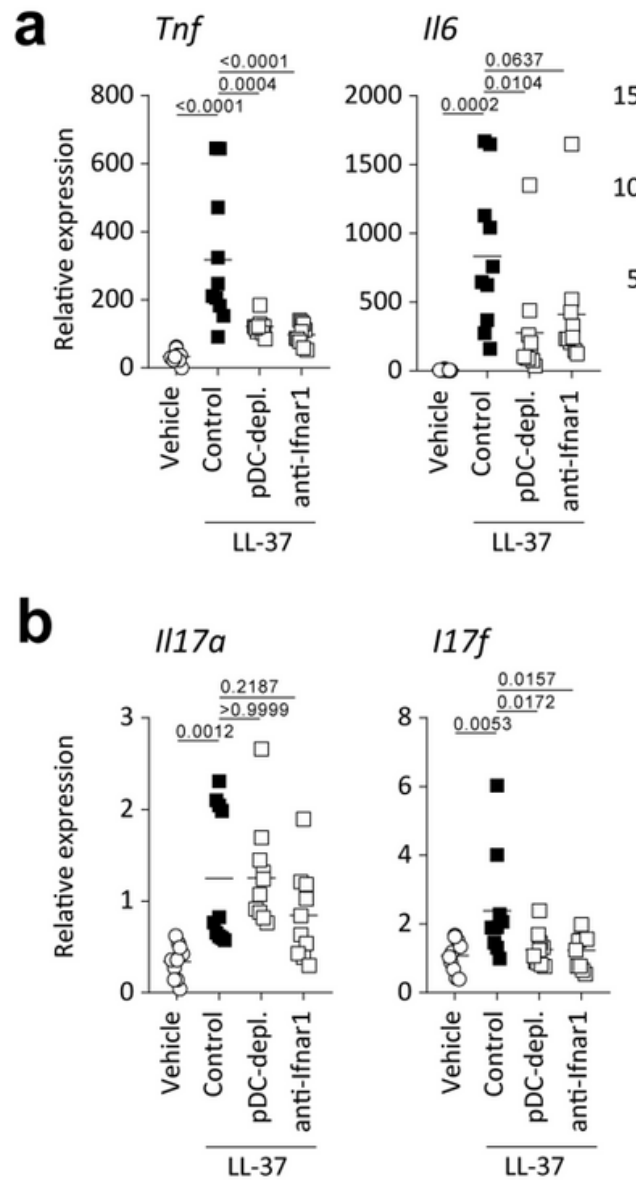

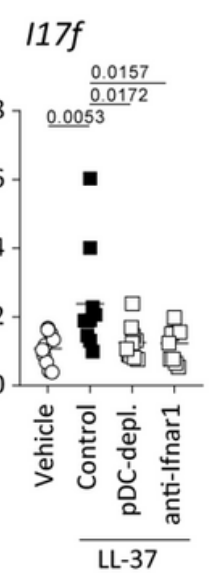

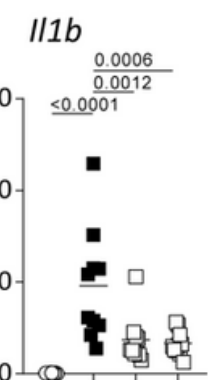
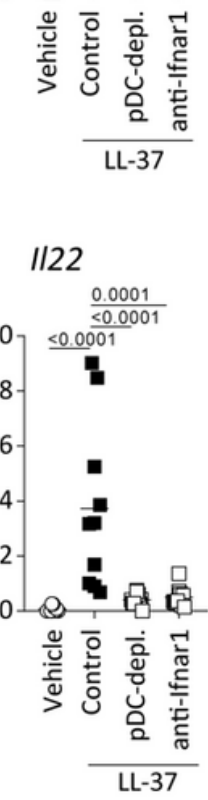
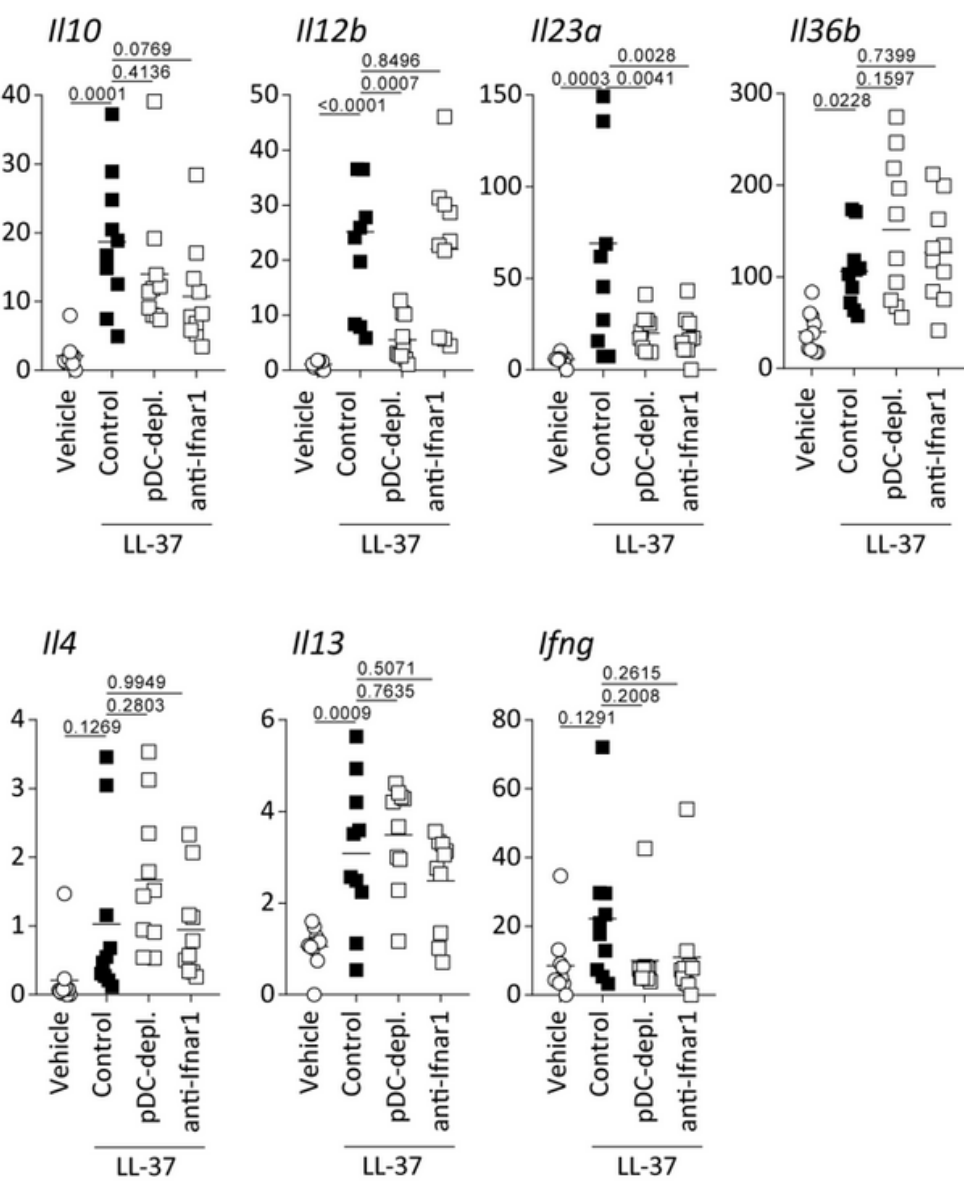

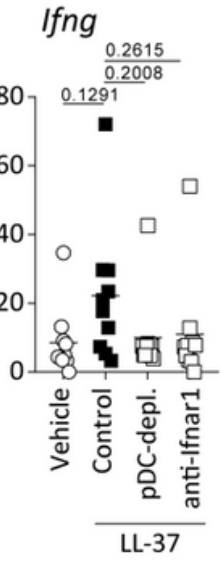

Figure 4

Type I interferon and pDC contribute to $\mathrm{TH} 1$ - and $\mathrm{TH} 22$-, but not $\mathrm{TH} 2$,-related inflammatory cytokine expression in situ. (a) Innate and (b) adaptive cytokine expression in biopsies obtained from intradermally injected mice with corresponding pre-treatments. Multiplicity adjusted P-values of one-way ANOVA are depicted. 

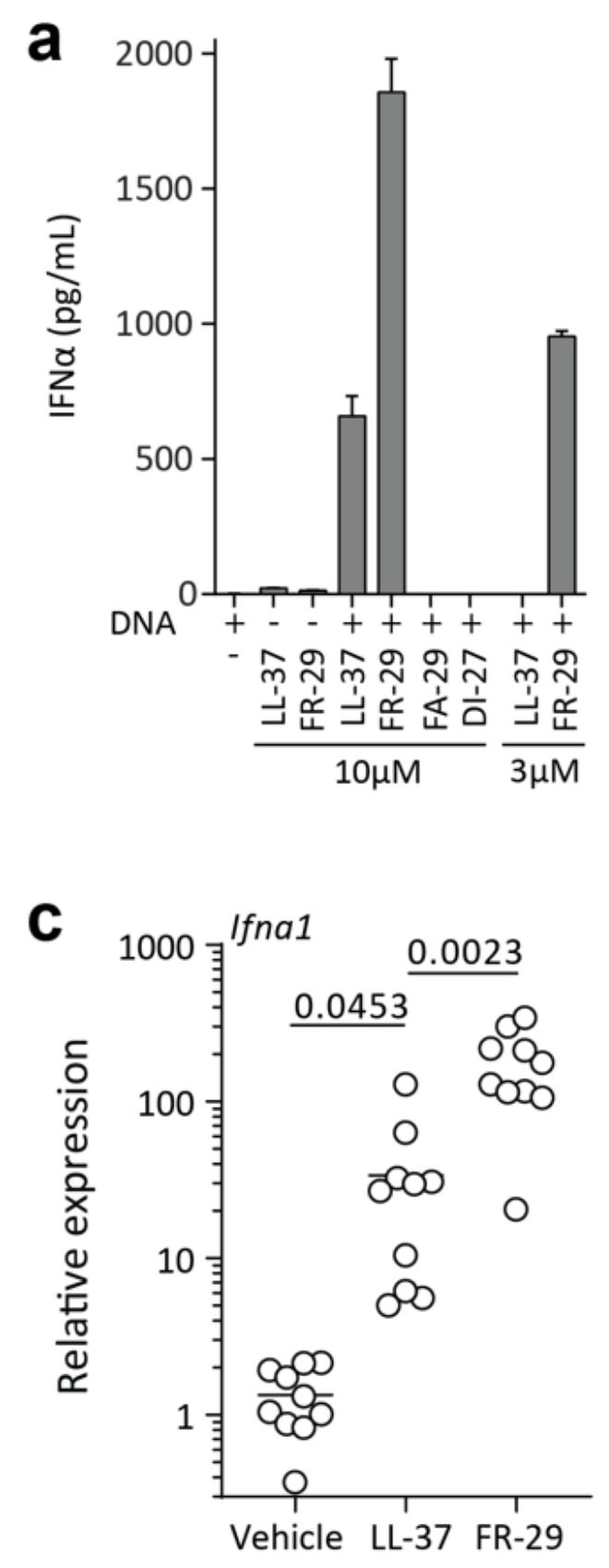

b

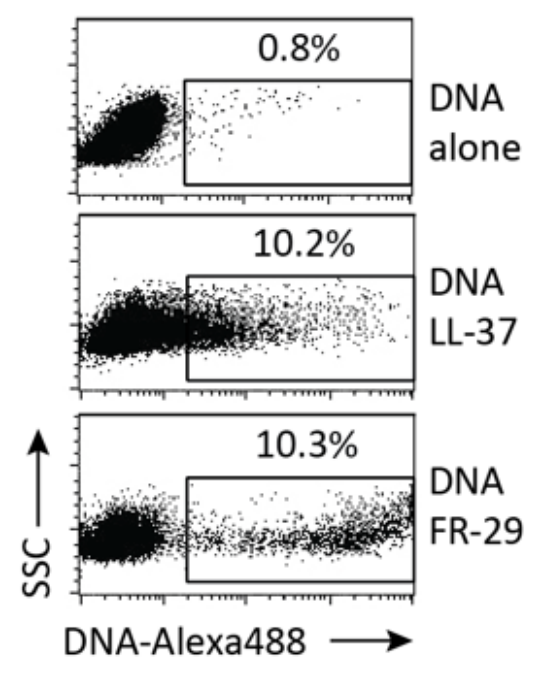

d

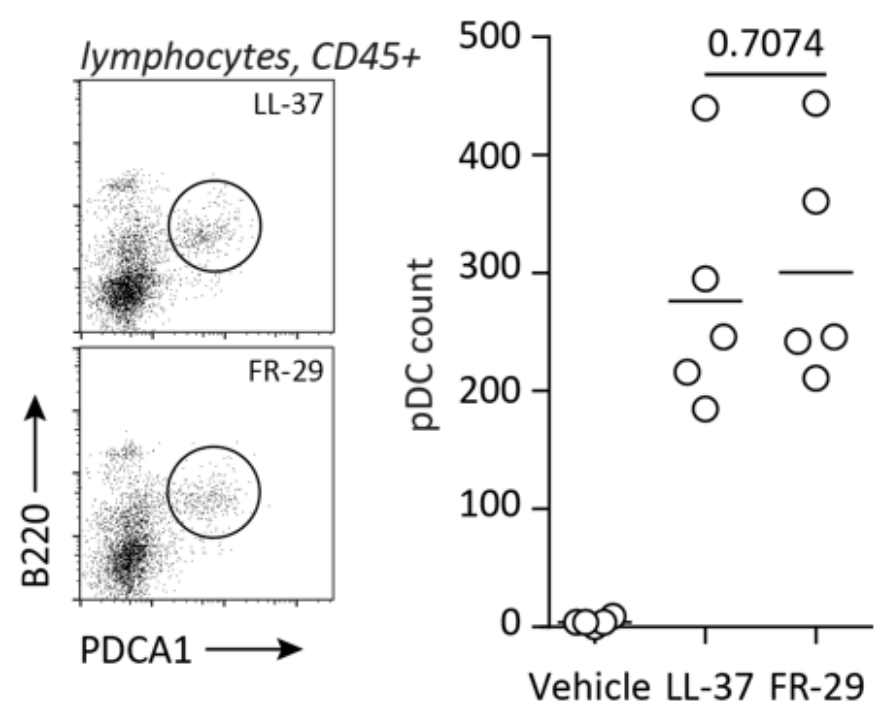

Figure 5

Cathelicidin peptide FR-29 activates pDCs more potently than LL-37 by binding DNA with more affinity, resulting in more potent internalisation of nucleic acids. (a) Isolated human pDCs were stimulated with the indicated cathelicidin peptides complexed with DNA, and IFNa was measured from supernatants after a 24h stimulation. (b) DNA was labelled with Alexa488 according to manufacturer's instructions, incubated with the indicated cathelicidin peptide, and put in culture with isolated human pDCs for $3 \mathrm{~h}$ before measuring the fluorescence by flow cytometry. (c) LL-37, or FR-29, or vehicle control (saline) were injected intradermally into wild-type Balb/c mice, and biopsies taken for gene expression analysis and (d) pDC quantification by flow cytometry. Multiplicity adjusted P-values of one-way ANOVA with Dunnett's multiple comparisons test are depicted in (c) and P-value of unpaired t test with Welch's correction in (d). 
a

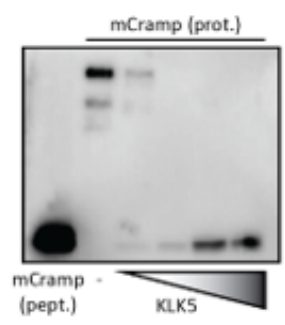

b
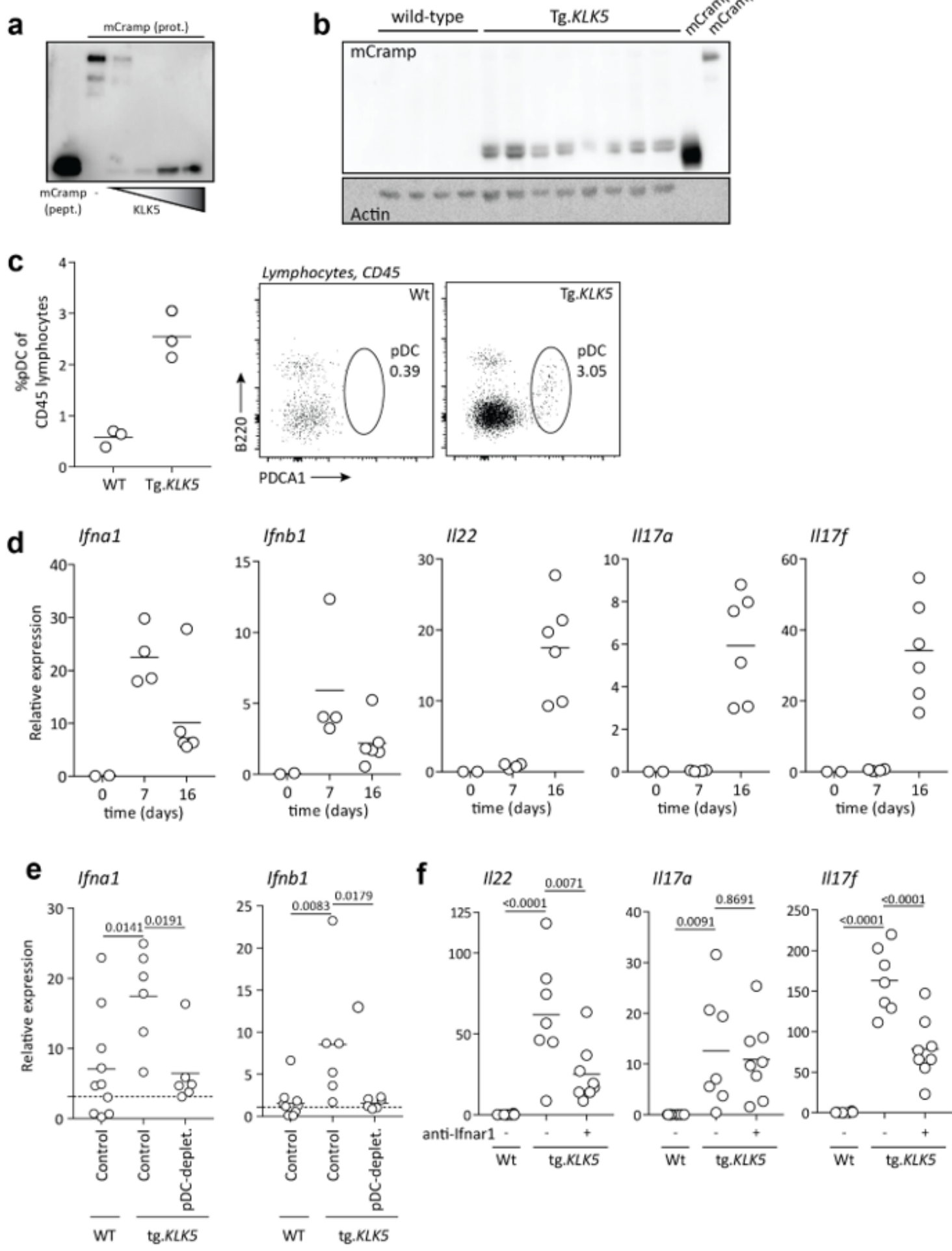

f $\quad 1122 \quad \underline{0.0071}$
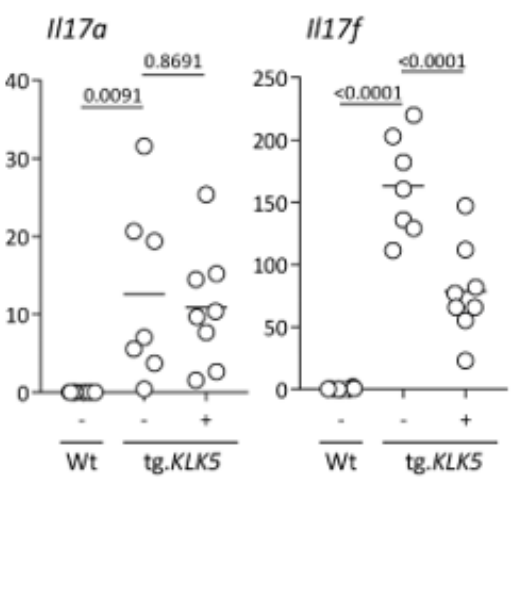

\section{Figure 6}

Kallikrein 5 overexpression in the epidermis results in pDC-derived overexpression of type I interferon and interferon-sustained II22 expression. (a) Murine Cramp recombinant protein (mCramp prot.) incubated with human KLK5 recombinant protein (hKLK5) for increasing times (5, 15, 30, and 90 minutes). Synthetic murine Cramp peptide (mCramp pept.) is used as a control for staining. (b) Skin samples from transgenic KLK5 mice (Tg.KLK5) and wild-type littermates was homogenised and probed for mCramp by 
western blot. Synthetic mCramp peptide and mCramp protein was used as a control for staining. (c) Skin lesions from 7-day old K5.KLK5 transgenic mice and the corresponding skin region from age-matched wild-type littermates was stained for pDCs by flow cytometry. (d) Gene expression from newborn, 7, and 16 day old transgenic K5.KLK5 mice. (e) Transgenic K5.KLK5 mice and wild-type littermates were depleted of pDCs from birth by sub-cutaneous injection of depleting antibodies, or injected with saline, every two days, and skin gene expression assessed. Results depicted are from two pooled experiments. (f) Transgenic K5.KLK5 and wild-type littermates were treated with anti-Ifnar antibodies, or injected with saline, every two days, and skin gene expression assessed. Results depicted are from two pooled experiments. Multiplicity adjusted P-values of one-way ANOVA with Dunnett's multiple comparisons test are depicted. 

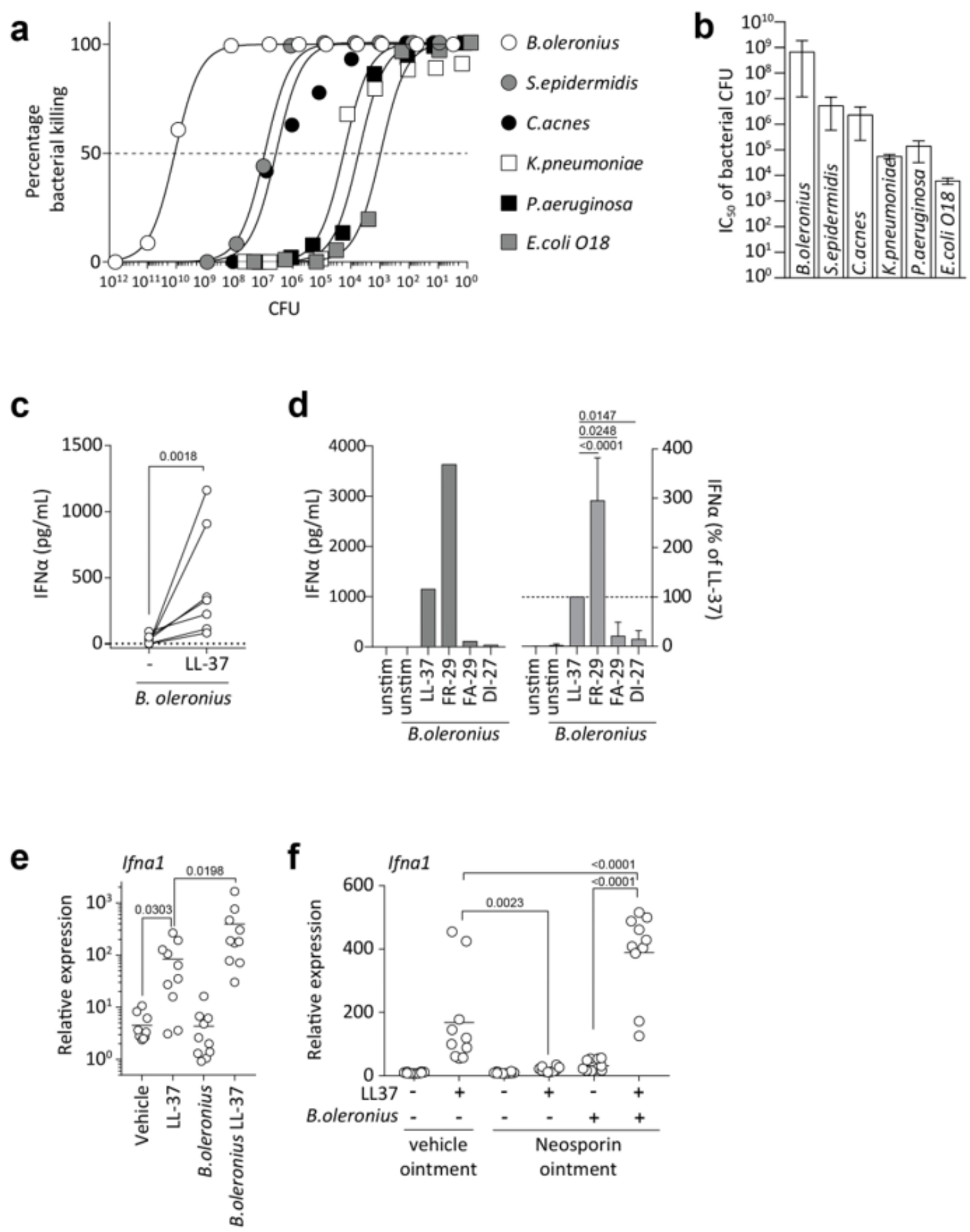

\section{Figure 7}

Cathelicidin-mediated killing of rosacea-associated bacteria activates pDCs to produce IFNa. (a) Bacteria at the indicated CFU were incubated with LL-37 at a constant $10 \mu \mathrm{M}$ concentration for $3 \mathrm{~h}$, and subsequently CFUs were counted after $18 \mathrm{~h}$ culture in their appropriate culture conditions following serial dilution. (b) CFU numbers efficiently killed with a constant concentration of LL-37 as in (a) and expressed as calculated IC50 values for each of the indicated bacteria (c) Plasmacytoid dendritic cells were isolated 
from human blood and stimulated with live B.oleronius, or B.oleronius pre-incubated with either LL-37 or left unstimulated (dotted line) for $24 \mathrm{~h}$, and IFNa production was assessed by ELISA. Results are pooled from 7 donours. (d) pDCs were stimulated as in (c) with either B.oleronius alone, or in pre-incubated with $5 \mu \mathrm{M}$ of the indicated cathelicidin peptide. Representative data from a single donor (left) and of at least three donors per condition expressed as percentage of each donors' LL-37 + B.oleronius condition. Pvalues of two-tailed paired t-test are depicted ( $*$ : $p<0.05 ; * \star$ : $p<0.01)$. (e) IFNa gene expression from biopsies collected from mice injected with LL-37, heat-killed B.oleronius, or heat-killed B.oleronius preincubated with LL-37, over the course of 24h. (f) Neosporin (containing Neomycin, Bacitracin, Polymyxin B) or vehicle ointment treated mice were injected intradermally over the course of $48 \mathrm{~h}$ with the indicated combinations of saline, LL-37, or LL-37 pre-incubated with B.oleronius. Results depicted in (c) and (d) are from two pooled experiments. P-values of two-tailed paired t-test are depicted in (c). Multiplicity adjusted P-values of one-way ANOVA are depicted in (d), (e), and (f). 

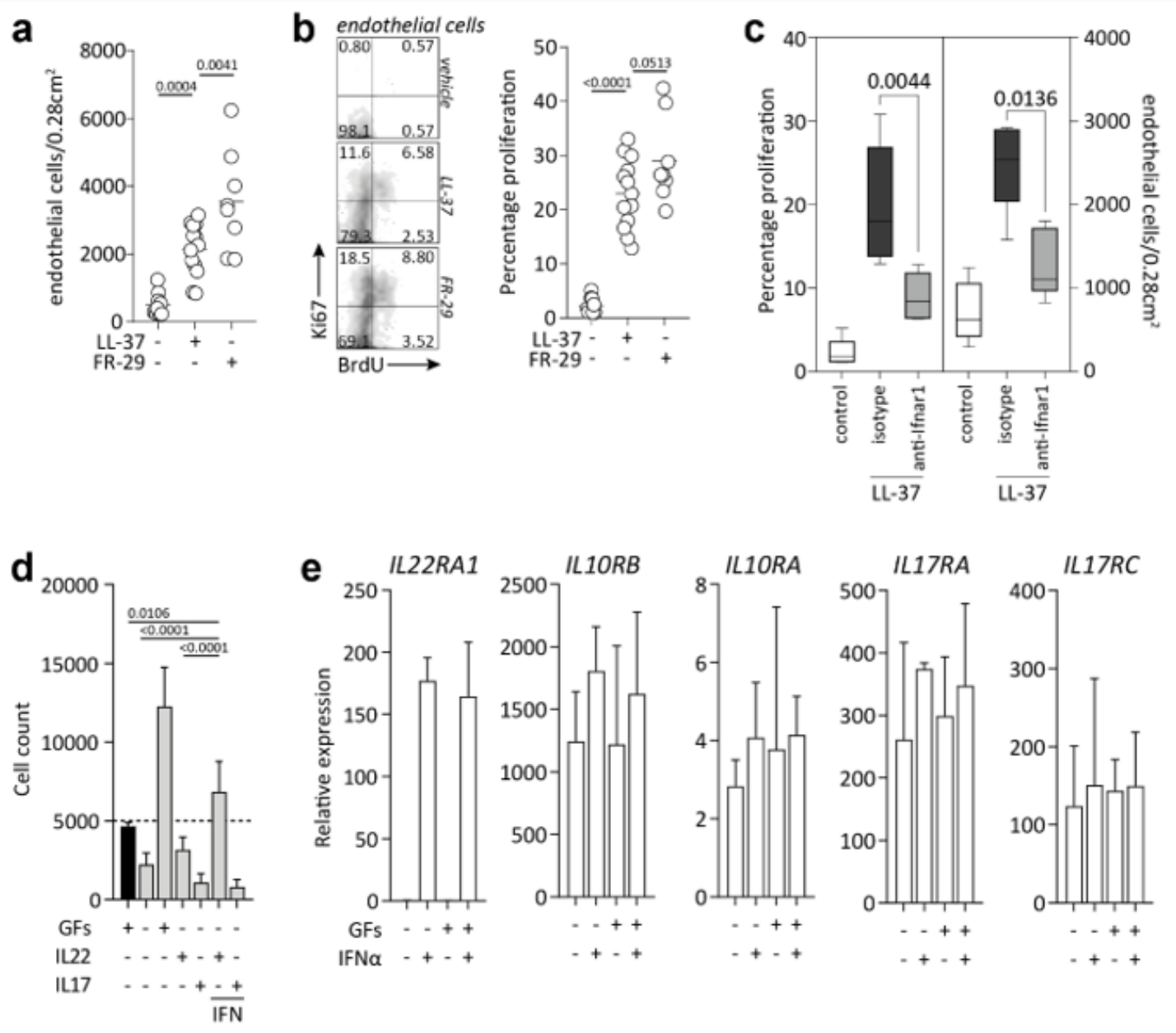

f

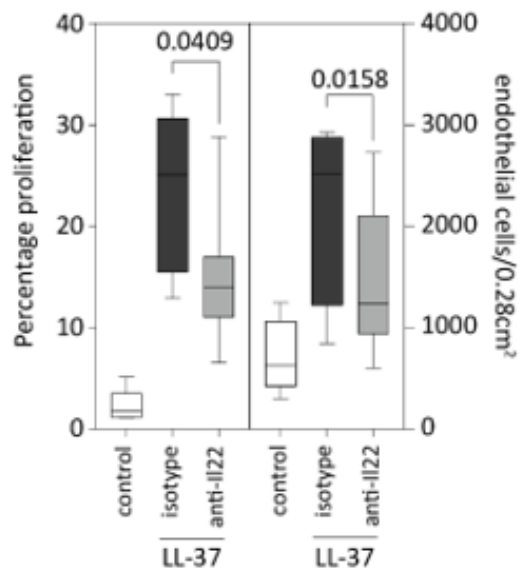

g

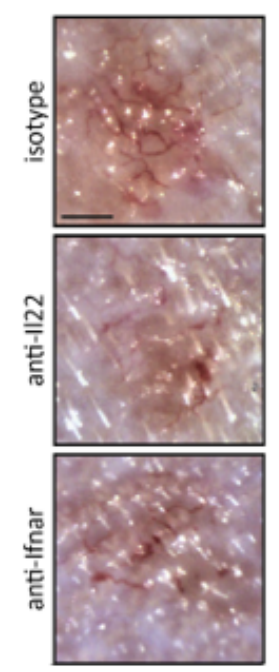

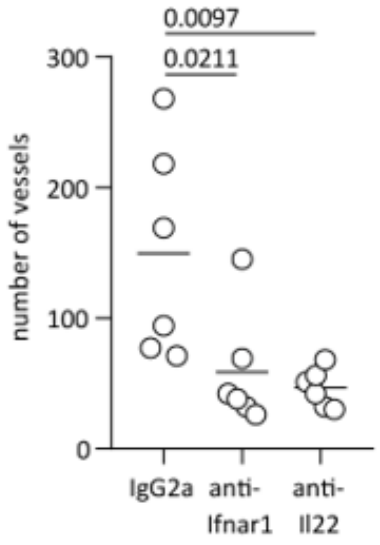

\section{Figure 8}

Type I interferon co-operates with IL22 in the induction of angiogenesis by promoting endothelial cell survival and proliferation. (a) The indicated cathelicidin peptides, or saline, were injected intradermally 4 times over a $48 \mathrm{~h}$ period as previously, followed by intraperitoneal injection with BrdU for $72 \mathrm{~h}$. Endothelial cell numbers were defined as $\mathrm{Cd} 31+\mathrm{Cd} 45$ - cells from debris-excluded pre-gating, and determined per injection site. (b) Proliferation was determined by staining of Ki67 and BrdU from cells gated on 
endothelial cells as in (A), and defined as Ki67-single, Ki67-BrdU-double, and BrdU-single positive cells. Results depicted are from three pooled experiments. (c) Mice intradermally injected with LL-37 or saline as in (a), (b) were either pre-treated with an anti-Ifnar blocking antibody or an IgG2a. Endothelial cell numbers and proliferation were determined as in (a), (b). Results depicted are representative of three experiments. (d) Human umbilical vein endothelial cells were plated (black bar at time of plating) and kept with or without growth factors (GFs) EGF, IGF1, FGF2, VEGFA, and with or without IL22 or IL17, in the presence or absence of IFNa. Live cells were defined as CytoGreen+CytoXOrange-cells and depicted as absolute counts. (e) Huvec were stimulated for $8 \mathrm{~h}$ in the indicated conditions, and gene expression analysed for the designated genes. Results are representative of three independent experiments. $(F)$ Mice were treated as in (c) and pre-treated with an anti-II22 blocking antibody or an IgG2a, and endothelial cell counts and proliferation was assessed as in (c). (g) Mice were treated as in (c) and (h), with either an IgG2a, anti-Ifnar, or anti-II22 antibody, and vessels visualised by videocapillaroscopy and counted in and around the injection site. Results depicted are from two pooled experiments. Multiplicity adjusted Pvalues of one-way ANOVA are depicted in (a), (b), (d), and (g). P-values of two-tailed Mann-Whitney nonparametric unpaired t-test are depicted in (c) and (f).

\section{Supplementary Files}

This is a list of supplementary files associated with this preprint. Click to download.

- SupportingInformation.docx 